\title{
Rest-to-rest attitude maneuvers and residual vibration reduction of a finite element model of flexible satellite by using input shaper
}

\author{
Setyamartana Parman* and Hideo Koguchi \\ ${ }^{a}$ Department of Mechanical Engineering, Nagaoka \\ University of Technology, Kamitomioka-machi \\ 1603-1, Nagaoka-shi, Niigata 940-2188, Japan
}

Received 16 January 1998

Revised 7 January 1999

A three-dimensional rest-to-rest attitude maneuver of flexible spacecraft equipped by on-off reaction jets is studied. Equations of motion of the spacecraft is developed by employing a hybrid system of coordinates and Lagrangian formulation. The finite element method is used to examine discrete elastic deformations of a particular model of satellite carrying flexible solar panels by modelling the panels as flat plate structures in bending. Results indicate that, under an unshaped input, the maneuvers induce undesirable attitude angle motions of the satellite as well as vibration of the solar panels. An input shaper is then applied to reduce the residual oscillation of its motion at several natural frequencies in order to get an expected pointing precision of the satellite. Once the shaped input is given to the satellite, the performance improves significantly.

Keywords: Euler angles, finite element, residual vibration, rest-to-rest, shaped input

\section{Introduction}

Precise orientation of spacecraft during its operation in space requires frequent corrections of its attitude. Attitude maneuver of rigid spacecraft can be done without a lot of vibration problems after reaching its desired attitude. For the flexible spacecraft maneuvering the attitude without regard to system flexibility or without controls on the flexible members, large amplitude transient and steady-state oscillations may oc-

\footnotetext{
${ }^{*}$ Corresponding author. Tel.: +81 258 471611, ext. 7475; Fax: +81 258 479770; E-mail: alamanda@stn.nagaokaut.ac.jp.
}

cur, especially when the system is equipped with onoff jets. Such a system often needs a rest-to-rest attitude maneuver with limited vibration both during and at the end of the maneuver. For example, it may be necessary to generate a torque profile such that the flexible spacecraft is rotated through a desired attitude angle, while the deflections of flexible members remain small throughout the maneuver and go to zero at the end of the maneuver.

To minimize modal vibration in a flexible spacecraft system, shaped torque command constructed from the finite trigonometric series has been proposed [15]. The method is implemented by convolving a sequence of impulses, an input shaper, with a desired system command to produce a shaped input which then is used to drive the system. The amplitudes and time locations of the impulses are determined by solving a set of constraint equations that attempt to control the dynamic response of the system.

Flexible spacecraft equipped with on-off reaction jets cannot produce the variable-amplitude actuation force that is usually required with input shaping; the spacecraft must be moved with constant amplitude force pulses. Some heuristic methods for extending input shaping to the case of on-off actuators have been developed [11]. The use of constraint on the impulse amplitudes have been used to generate time-optimal command profiles for on-off reaction jets. A lot of works $[8,12,17]$ demonstrated on-off input shaping with mass, spring, and damper simulations. These works have concentrated on eliminating residual vibration. No constraints were placed on the amplitude of deflection during the slew. Input shaping is very successful at eliminating residual vibration and has the benefit of decreasing transient deflection when compared to bang-bang control [10].

A spacecraft or a satellite in operation needs certain accuracies in its attitude. The KOREASAT requires a satellite with an antenna beam pointing error 
not greater than $0.07^{\circ}$ in roll and pitch, and not more than $0.2^{\circ}$ in yaw [3]. To satisfy such kind of criteria, the flexible satellite maneuvered by shaped inputs must have residual attitude oscillation must be less than the permissible maximum error. However, for the system with a large number of flexible modes such as the finite element model of satellite with flexible solar panels studied by Koguchi and Parman [4-6], when the satellite is subjected to a shaped input suppressing residual vibration at the frequency with largest vibration amplitude, other frequencies can amplify the resulted residual vibration so that the satellite attitude oscillation after maneuver is still greater than the permissible attitude error. In this case, shaped inputs suppressing residual vibration at several frequencies until the satellite attitude oscillation are less than its pointing accuracy are needed.

This paper presents computer simulations of rest-torest attitude maneuvers of a satellite with flexible solar panels under shaped inputs. The equation of motion is derived by using a hybrid system of coordinates and a Lagrange's formulation, and the finite element method is used to discrete elastic deformations of solar panels by modelling them as flat plate structures in bending, such as the model proposed by Koguchi and Parman [4-6]. The shaped input is selected to make zero vibration on the frequency with the greatest amplitude of residual attitude oscillation and small vibration at several other frequencies in order to get the permissible residual attitude oscillation of satellite. The maximum deflections of solar panels during the attitude maneuver are also studied qualitatively in order to find the strategy in choosing an input shaper with small deflection of flexible members.

\section{Formulation of the equations of motion}

To formulate the equations of motion of flexible spacecraft in space, a system of reference frames to measure motions of each structural subsystem needs to be determined. Factors that will affect the choice of reference frames are: (i) the simplicity in expressing the potential and kinetic energies; (ii) the simplicity in formulating the equations of motion; (iii) the simplicity of constraint equations relating to the orientation of the frame relative to the structural subsystem; and (iv) the ability to separate equations governing the spacecraft's rigid body motions from the motions of elastic deformations of flexible substructures.

The particular spacecraft being studied in this paper is a gravity oriented-stabilized satellite containing a rigid main body and two symmetrical flexible solar panels, such as the Canadian Communications Technology Satellite, in a circular orbit. Environmental drags to the satellite during orbiting the earth are supposed to be absent or can be neglected. As in a lot of works in flexible spacecraft dynamics [7,9], a hybrid system of coordinates is used in this paper. As discrete coordinates to express unrestricted motions of the rigid main body, the inertial reference frame, the orbital reference frame, and the rigid main body fixed reference frame are defined as shown in Fig. 1;

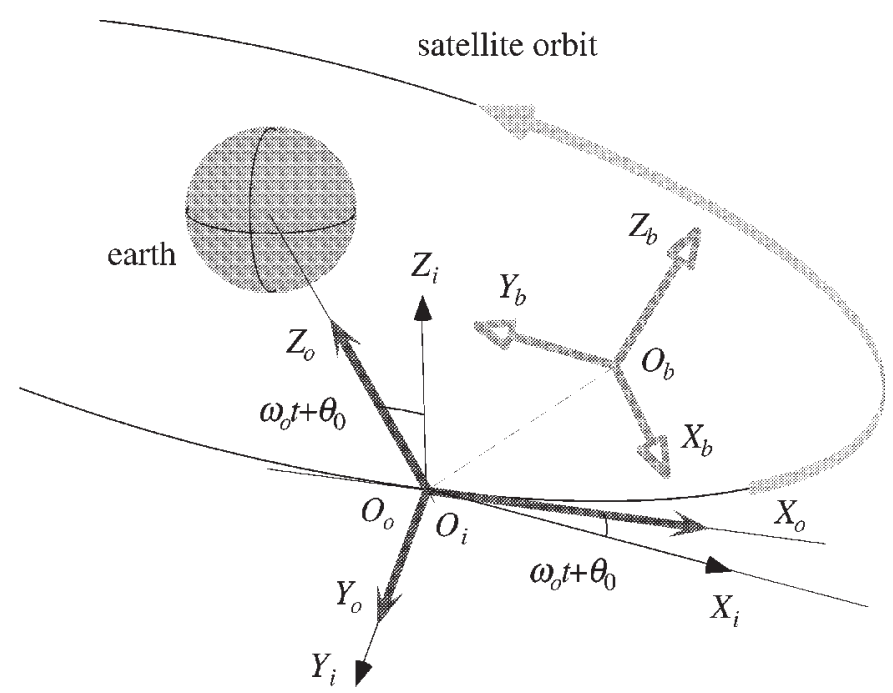

Fig. 1. The inertial reference frame $F_{i}\left(O_{i} X_{i} Y_{i} Z_{i}\right)$, orbital reference frame $F_{o}\left(O_{o} X_{o} Y_{o} Z_{o}\right)$, and main body fixed reference frame $F_{b}\left(O_{b} X_{b} Y_{b} Z_{b}\right)$ definitions. 
while as distributed coordinates to describe the flexible substructure time-varying deformations, the flexible substructure local reference frames are used. The inertial reference frame is fixed with respect to the stars, and referred to for all unrestricted dynamic motions of the satellite. In this paper, the inertial reference frame $F_{i}\left(O_{i} X_{i} Y_{i} Z_{i}\right)$ is defined as follows: (i) the origin of the inertial reference frame, $O_{i}$, is selected at a point of its nominal orbit, (ii) the first axis, $X_{i}$, is directed along a fixed celestial direction in the orbit plane, (iii) the second axis, $Y_{i}$, is normal to the orbit plane, (iv) and the third axis, $Z_{i}$, completes the orthogonal set, i.e., in the orbit plane. The orbital reference frame, $F_{o}\left(O_{o} X_{o} Y_{o} Z_{o}\right)$, is defined as follows: (i) the origin of the orbital reference frame $O_{o}$ is located on the nominal satellite orbit, (ii) the first axis, $X_{o}$, points to the orbital direction, (iii) the second axis, $Y_{o}$, is normal to the orbit plane, (iv) and the third axis, $Z_{o}$, points to the centre of earth. The orbital reference frame can be obtained from the inertial reference frame by rotation $\omega_{o} t+\theta_{0}$ along $Y_{i}$-axis, where $\omega_{o}$ is the orbital angular velocity, and $\theta_{0}$ is a constant angle. The rigid main body reference frame, $F_{b}\left(O_{b} X_{b} Y_{b} Z_{b}\right)$, is assumed to be fixed on the rigid main body, and flexible substructure reference frames are assumed to be fixed on each substructure. The orientation of the rigid main body fixed frame relative to the orbital reference frame is expressed in Euler angles: roll $(\phi)$, pitch $(\theta)$, and yaw $(\psi)$; where the definition of these angles can be seen in Fig. 2. From Fig. 2, the transformation from $F_{o}$ to $F_{b}$ is accomplished by premultiplication with the transformation matrix $\mathbf{T}_{o, b}$, which is

$$
\mathbf{T}_{o, b}=\left[\begin{array}{ccc}
c \theta c \psi & c \theta s \psi & -s \theta \\
s \phi s \theta c \psi-c \phi s \psi & s \phi s \theta s \psi+c \phi c \psi & s \phi c \theta \\
c \phi s \theta c \psi+s \phi s \psi & c \phi s \theta s \psi-s \phi c \psi & c \phi c \theta
\end{array}\right],
$$

where $s$ and $c$ in the above equation express the sine and cosine functions respectively. The rigid body frame and substructure frames $F_{l}\left(O_{l} X_{l} Y_{l} Z_{l}\right)$ are shown together in Fig. 3. All reference frames in this paper follow the right-hand-side orthogonal system.

The satellite being observed for study carries two symmetrical flexible solar panels, as shown in Fig. 3, whose longitudinal axis is in the $Y_{b}$-axis direction of the main body. For the finite element application, each solar panel is divided into 16 rectangular bending plate elements. The elements on the right-side are numbered from 1 through 16 and on the left-side are from 17 through 32, while their nodal points are numbered from 1 through 27 and from 28 through 54. Substructure reference frames on the left-side have the same normal directions ( $Z_{l}$-axes) to the solar panel's plane but the reverse in-plane directions ( $X_{l}$ and $Y_{l}$-axes) with the frames on the right-side. Both the $Y_{b}$-axis of rigid body and $Y_{l}$-axes of right-side substructure are in the same direction. The origin of rigid body frame $O_{b}$ is placed on the midpoint of the longitudinal axis of solar panels. Solar panels are oriented towards the sun, and the declination with respect the $X_{b}$-axis of rigid body frame is identified by the offset angle $\delta$.

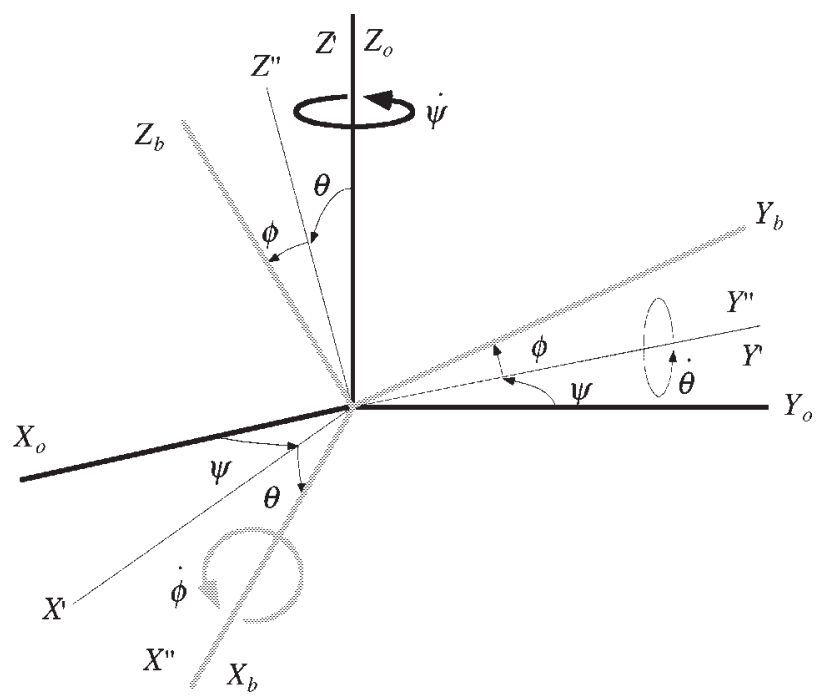

Fig. 2. The transformation from the orbital reference frame $F_{o}$ to the main body fixed reference frame $F_{b}$. 


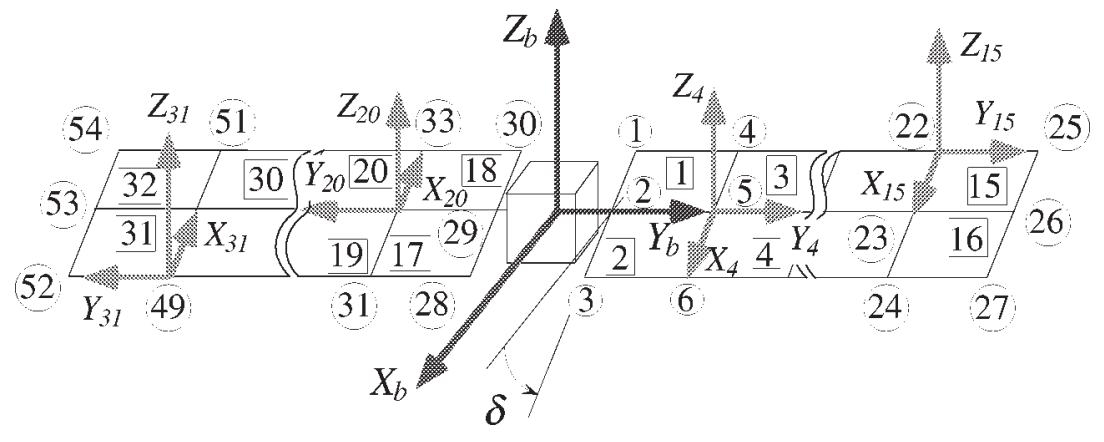

Fig. 3. The model of satellite being investigated; element numbering, nodal point numbering, rigid main body fixed reference frame, and local reference frames.

\subsection{General equations of motion of flexible spacecraft}

In this section, the mathematical model of general gravity oriented and nonspinning flexible spacecraft dynamics will be derived by using a Lagrangian formulation, so that the expressions of kinetic energy and potential energy for the whole spacecraft need to be determined first. The spacecraft considered here consists of a rigid main body and several flexible substructures attached to it. For this configuration, both the kinetic energy and potential energy of spacecraft can be determined by analyzing the rigid body and flexible structural subsystems separately, then summing kinetic energies and potential energies to obtain the total energy.

\subsubsection{Kinetic energy}

To formulate the kinetic energy of the spacecraft, we must consider firstly the kinetic energy of the particle $p$ with mass $\mathrm{d} m$ relative to the inertial reference frame, which can be written as

$$
\mathrm{d} E_{k}=\frac{1}{2} \dot{\mathbf{r}}_{i, p}^{T} \dot{\mathbf{r}}_{i, p} \mathrm{~d} m
$$

where $\mathbf{r}_{i, p}$ is a vector from the origin of inertial reference frame to the particle $p$, and the overdot means the differentiation with respect to time relative to the inertial reference frame.

For particle $p$ in the rigid main body, $\mathbf{r}_{i, p}$ can be expressed as

$$
\mathbf{r}_{i, p}=\mathbf{r}_{i, b}+\mathbf{r}_{b, p}
$$

with $\dot{\mathbf{r}}_{i, p}$ is

$$
\dot{\mathbf{r}}_{i, p}=\dot{\mathbf{r}}_{i, b}+{ }^{b} \dot{\mathbf{r}}_{b, p}+\boldsymbol{\omega}_{b, i} \times{ }^{b} \mathbf{r}_{b, p},
$$

where $\mathbf{r}_{i, b}$ is a vector from $O_{i}$ to $O_{b},{ }^{b} \mathbf{r}_{b, p}$ is a vector from $O_{b}$ to the particle $p$ being expressed in $F_{b}, \boldsymbol{\omega}_{b, i}$ is the angular velocity vector of $F_{b}$ relative to $F_{i}$, and ${ }^{b} \dot{\mathbf{r}}_{b, p}$ is the differentiation of $\mathbf{r}_{b, p}$ with respect to time measured in $F_{b}$. By using Eq. (4) in (2) and integrating will result the kinetic energy of the rigid body of spacecraft in the following form:

$$
E_{k_{b}}=\frac{1}{2} \dot{\mathbf{r}}_{i, b}^{T} \dot{\mathbf{r}}_{i, b} m_{b}+\frac{1}{2} \boldsymbol{\omega}_{b, i}^{T} \mathbf{I}_{b} \boldsymbol{\omega}_{b, i}+\dot{\mathbf{r}}_{i, b}^{T} \mathbf{Q}_{b} \boldsymbol{\omega}_{b, i}
$$

where $m_{b}$ is the total mass of the rigid main body, $\mathbf{I}_{b}$ is the inertia matrix of the main body relative to the origin of the main body fixed frame $O_{b}$ :

$$
\mathbf{I}_{b}=\int_{m_{b}}{ }^{b} \tilde{\mathbf{r}}_{b, p}^{T}{ }^{b} \tilde{\mathbf{r}}_{b, p} \mathrm{~d} m
$$

since ${ }^{b} \tilde{\mathbf{r}}_{b, p}$ is the skew symmetric matrix of ${ }^{b} \mathbf{r}_{b, p}$, and $\mathbf{Q}_{b}$ is the coupling matrix between translational and rotational displacements of the rigid main body:

$$
\mathbf{Q}_{b}=\int_{m_{b}}{ }^{b} \tilde{\mathbf{r}}_{b, p}^{T} \mathrm{~d} m .
$$

If the origin of rigid main body fixed frame coincides with the mass centre of the main body, the value of $\mathbf{Q}_{b}$ is equal to zero.

For particle $p$ in a flexible substructure, from Fig. 4, $\mathbf{r}_{i, p}$ can be expressed in the following form:

$$
\mathbf{r}_{i, p}=\mathbf{r}_{i, b}+\mathbf{r}_{b, o}+\mathbf{r}_{o, p_{0}}+\mathbf{r}_{p_{0}, p}
$$

with $\dot{\mathbf{r}}_{i, p}$ in the following expression:

$$
\begin{aligned}
\dot{\mathbf{r}}_{i, p}= & \dot{\mathbf{r}}_{i, b}+{ }^{b} \dot{\mathbf{r}}_{b, o}+{ }^{b} \dot{\mathbf{r}}_{o, p_{0}}+{ }^{b} \dot{\mathbf{r}}_{p_{0}, p} \\
& +\boldsymbol{\omega}_{b, i} \times\left({ }^{b} \mathbf{r}_{b, o}+{ }^{b} \mathbf{r}_{o, p_{0}}+{ }^{b} \mathbf{r}_{p_{0}, p}\right),
\end{aligned}
$$

where $\mathbf{r}_{b, o}$ is a vector from $O_{b}$ to an origin of flexible substructure local reference frame $O_{l}, \mathbf{r}_{o, p_{0}}$ is a vector 


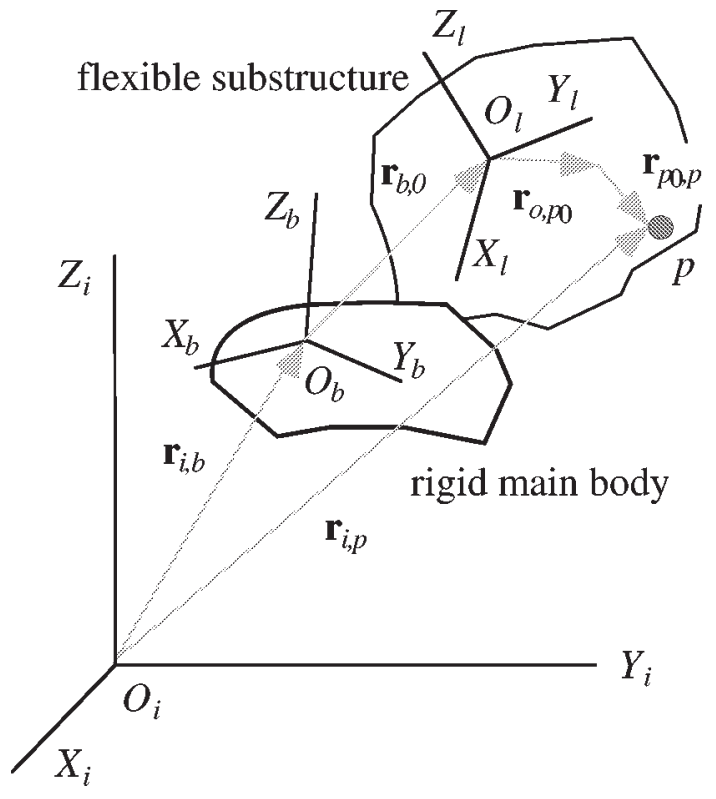

Fig. 4. Particle $p$ in a flexible substructure.

from $O_{l}$ to the observed particle $p$ in the undeformed state of substructure, $\mathbf{r}_{p_{0}, p}$ is the deformation vector of particle $p,{ }^{b} \dot{\mathbf{r}}_{b, o},{ }^{b} \dot{\mathbf{r}}_{o, p_{0}}$, and ${ }^{b} \dot{\mathbf{r}}_{p_{0}, p}$ are the differentiations of $\mathbf{r}_{b, o}, \mathbf{r}_{o, p_{0}}$ and $\mathbf{r}_{p_{0}, p}$ with respect to time measured in $F_{b}$, and ${ }^{b} \mathbf{r}_{b, o},{ }^{b} \mathbf{r}_{o, p_{0}}$, and ${ }^{b} \mathbf{r}_{p_{0}, p}$ are $\mathbf{r}_{b, o}, \mathbf{r}_{o, p_{0}}$ and $\mathbf{r}_{p_{0}, p}$ being expressed in $F_{b}$.

The deformation vector of particle $p, \mathbf{r}_{p_{0}, p}$, for simplicity in expression and efficiency in numerical calculation, is expressed in the substructure local reference frame. If the orientation and position of the undeformed state of flexible substructures relative to the rigid main body does not vary with respect to time, i.e., the angular velocity of $F_{l}$ relative to $F_{b}$ is equal to zero, Eq. (9) then can be expressed as

$$
\begin{aligned}
\dot{\mathbf{r}}_{i, p}= & \dot{\mathbf{r}}_{i, b}+\mathbf{T}_{b, l}^{T}{ }^{l} \dot{\mathbf{r}}_{p_{0}, p}+\boldsymbol{\omega}_{b, i} \\
& \times \mathbf{T}_{b, l}^{T}\left({ }^{l} \mathbf{r}_{b, o}+{ }^{l} \mathbf{r}_{o, p_{0}}+{ }^{l} \mathbf{r}_{p_{0}, p}\right),
\end{aligned}
$$

where $\mathbf{T}_{b, l}$ is the transformation matrix from $F_{b}$ to $F_{l}$.

Let us consider that flexible substructures are represented by $N$ elastic elements. Employing Eq. (10), the integration of Eq. (2) for the element $j(j=1,2$, $\ldots, N)$ of flexible substructure for small deformations will result

$$
\begin{aligned}
E_{k a_{j}}= & \frac{1}{2} \dot{\mathbf{r}}_{i, b}^{T} \dot{\mathbf{r}}_{i, b} m_{j}+\frac{1}{2} \int_{m_{j}}{ }^{l} \dot{\mathbf{r}}_{p_{0}, p}^{T}{ }^{l} \dot{\mathbf{r}}_{p_{0}, p} \mathrm{~d} m \\
& +\frac{1}{2} \boldsymbol{\omega}_{b, i}^{T} \mathbf{T}_{b, l}^{T} \int_{m_{j}}\left({ }^{l} \tilde{\mathbf{r}}_{b, o}+{ }^{l} \tilde{\mathbf{r}}_{o, p_{0}}\right)^{T}
\end{aligned}
$$

$$
\begin{aligned}
& \times\left({ }^{l} \tilde{\mathbf{r}}_{b, o}+{ }^{l} \tilde{\mathbf{r}}_{o, p_{0}}\right) \mathrm{d} m \mathbf{T}_{b, l} \boldsymbol{\omega}_{b, i} \\
& +\boldsymbol{\omega}_{b, i}^{T} \mathbf{T}_{b, l}^{T} \int_{m_{j}}\left({ }^{l} \tilde{\mathbf{r}}_{b, o}+{ }^{l} \tilde{\mathbf{r}}_{o, p_{0}}\right){ }^{l} \dot{\mathbf{r}}_{p_{0}, p} \mathrm{~d} m \\
& +\dot{\mathbf{r}}_{i, b}^{T} \mathbf{T}_{b, l}^{T} \int_{m_{j}}\left({ }^{l} \tilde{\mathbf{r}}_{b, o}+{ }^{l} \tilde{\mathbf{r}}_{o, p_{0}}\right)^{T} \mathrm{~d} m \mathbf{T}_{b, l} \boldsymbol{\omega}_{b, i} \\
& +\dot{\mathbf{r}}_{i, b}^{T} \mathbf{T}_{b, l}^{T} \int_{m_{j}}{ }^{l} \dot{\mathbf{r}}_{p_{0}, p} \mathrm{~d} m
\end{aligned}
$$

where ${ }^{l} \tilde{\mathbf{r}}_{b, o}$ and ${ }^{l} \tilde{\mathbf{r}}_{o, p_{0}}$ are skew symmetric matrices of ${ }^{l} \mathbf{r}_{b, o}$ and ${ }^{l} \mathbf{r}_{o, p_{0}}$ respectively, and $m_{j}$ is the mass of element $j$. It should be noted that all integrations in Eq. (11) are extended over the undeformed state which serves as the reference state of element $j$.

By following the finite element method [18], ${ }^{l} \mathbf{r}_{p_{0}, p}$ in the $j$-th element can be expressed in the following form:

$$
{ }^{l} \mathbf{r}_{p_{0}, p}(x, y, z)=\mathbf{C}_{j}(x, y, z) \mathbf{d}_{j},
$$

where $\mathbf{C}_{j}$ is a shape function matrix of the element $j$, and $\mathbf{d}_{j}$ is the displacement vector of the element $j$. The total kinetic energy of flexible substructures then can be produced in the following form:

$$
\begin{aligned}
E_{k a}= & \sum_{j=1}^{N} E_{k a_{j}} \\
= & \frac{1}{2} \dot{\mathbf{r}}_{i, b}^{T} \dot{\mathbf{r}}_{i, b} m_{a}+\frac{1}{2} \dot{\mathbf{d}}^{T} \mathbf{M} \dot{\mathbf{d}}+\frac{1}{2} \boldsymbol{\omega}_{b, i}^{T} \mathbf{I}_{a} \boldsymbol{\omega}_{b, i} \\
& +\boldsymbol{\omega}_{b, i}^{T} \mathbf{A} \dot{\mathbf{d}}+\dot{\mathbf{r}}_{i, b}^{T} \mathbf{W} \dot{\mathbf{d}}+\dot{\mathbf{r}}_{i, b}^{T} \mathbf{Q}_{a} \boldsymbol{\omega}_{b, i},
\end{aligned}
$$

where it has been used in Eq. (13) definitions of the total mass of flexible substructures:

$$
m_{a}=\sum_{j=1}^{N} m_{j}
$$

the total mass matrix of flexible substructures:

$$
\mathbf{M}=\sum_{j=1}^{N} \mathbf{P}_{j}^{T} \mathbf{M}_{j} \mathbf{P}_{j},
$$

the total inertia matrix of flexible substructures:

$$
\mathbf{I}_{a}=\sum_{j=1}^{N} \mathbf{T}_{j}^{T} \mathbf{I}_{j} \mathbf{T}_{j},
$$


the total coupling matrix for the rotational displacements of the rigid main body and the displacements of flexible substructures:

$$
\mathbf{A}=\sum_{j=1}^{N} \mathbf{T}_{j}^{T} \mathbf{A}_{j} \mathbf{P}_{j}
$$

the total coupling matrix for the translational displacements of the rigid main body and the displacements of flexible substructures:

$$
\mathbf{W}=\sum_{j=1}^{N} \mathbf{T}_{j}^{T} \mathbf{W}_{j} \mathbf{P}_{j}
$$

and the total coupling matrix for the translational and rotational displacements of the rigid main body:

$$
\mathbf{Q}_{a}=\sum_{j=1}^{N} \mathbf{T}_{j}^{T} \mathbf{Q}_{j} \mathbf{T}_{j}
$$

while

$$
\begin{aligned}
& \mathbf{M}_{j}=\int_{m_{j}} \mathbf{C}_{j}^{T} \mathbf{C}_{j} \mathrm{~d} m, \\
& \mathbf{I}_{j}=\int_{m_{j}}\left({ }^{l} \tilde{\mathbf{r}}_{b, o}+{ }^{l} \tilde{\mathbf{r}}_{o, p_{0}}\right)^{T}\left({ }^{l} \tilde{\mathbf{r}}_{b, o}+{ }^{l} \tilde{\mathbf{r}}_{o, p_{0}}\right) \mathrm{d} m, \\
& \mathbf{A}_{j}={ }^{l} \tilde{\mathbf{r}}_{b, o} \mathbf{W}_{j}+\int_{m_{j}}{ }^{l} \tilde{\mathbf{r}}_{o, p_{0}} \mathbf{C}_{j} \mathrm{~d} m, \\
& \mathbf{W}_{j}=\int_{m_{j}} \mathbf{C}_{j} \mathrm{~d} m, \\
& \mathbf{Q}_{j}=\int_{m_{j}}\left({ }^{l} \tilde{\mathbf{r}}_{b, o}+{ }^{l} \tilde{\mathbf{r}}_{o, p_{0}}\right)^{T} \mathrm{~d} m,
\end{aligned}
$$

$\mathbf{T}_{j}$ is $\mathbf{T}_{b, l}$ for the $j$-th element, and $\mathbf{P}_{j}$ is the assembling matrix relating the $j$-th element displacement vector $\mathbf{d}_{j}$ and the displacement vector of total flexible substructures $\mathbf{d}$ as expressed in the following form:

$$
\mathbf{d}_{j}=\mathbf{P}_{j} \mathbf{d} .
$$

\subsubsection{Potential energy}

The potential energy of the satellite consists of the potential energy of its undeformed state and the potential energy due to elastic deformations of flexible substructures. The potential energy of the undeformed state, in this research, is measured relative to the earth

$$
E_{p r}=E_{p r}\left(\mathbf{r}_{e, b}\right),
$$

where $\mathbf{r}_{e, b}=\mathbf{r}_{e, i}+\mathbf{r}_{i, b}, \mathbf{r}_{e, i}$ is a vector from the centre of the earth to $O_{i}$. If the satellite orbit is circular, $\mathbf{r}_{e, i}$ is constant, so

$$
E_{p r}=E_{p r}\left(\mathbf{r}_{i, b}\right)
$$

The potential energy due to elastic deformations is the sum of strain energy of flexible substructures and the potential energy due to external forces acting on the substructures with minus sign. The potential energy of element $j(j=1,2, \ldots, N)$ in the flexible substructure can be written as [18]:

$$
E_{p a_{j}}=\frac{1}{2} \int_{V_{j}} \boldsymbol{\sigma}_{j}^{T} \varepsilon_{j} \mathrm{~d} V-\int_{V_{j}}{ }^{l} \mathbf{r}_{p_{0}, p}^{T} \boldsymbol{F}_{f} \mathrm{~d} V
$$

where $\boldsymbol{\sigma}_{j}, \boldsymbol{\varepsilon}_{j}$, and $\boldsymbol{F}_{f}$ are stress vector, strain vector, and distributed external forces vector at the element $j$. Again, by following the general finite element method procedures and employing Eq. (12), the total potential energy due to the elastic deformations of flexible substructures can be written in the following form:

$$
E_{p a}=\frac{1}{2} \mathbf{d}^{T} \mathbf{K d}-\mathbf{d}^{T} \boldsymbol{F}_{a},
$$

where

$$
\mathbf{K}=\sum_{j=1}^{N} \mathbf{P}_{j}^{T} \mathbf{K}_{j} \mathbf{P}_{j}
$$

is the total stiffness matrix of flexible substructures,

$$
\boldsymbol{F}_{a}=\sum_{j=1}^{N} \mathbf{P}_{j}^{T} \boldsymbol{F}_{j}
$$

is the total discrete external forces vector acting on all nodes of flexible substructures, while

$$
\boldsymbol{F}_{j}=\int_{V_{j}} \mathbf{C}_{j}^{T} \boldsymbol{F}_{f} \mathrm{~d} V
$$

is a discrete external forces acting on the nodes of element $j$,

$$
\mathbf{K}_{j}=\int_{V_{j}} \mathbf{C}_{j}^{T} \mathbf{B}_{j}^{T} \mathbf{R}_{j} \mathbf{B}_{j} \mathbf{C}_{j} \mathrm{~d} V
$$


is a stiffness matrix of element $j, \mathbf{B}_{j}$ is an operator matrix containing first or second order derivative operators, and $\mathbf{R}_{j}$ is an elasticity matrix of element $j$.

\subsubsection{Lagrangian formulation}

To derive the general equations of motion of flexible spacecraft by using Lagrangian procedure, the Lagrangian operator

$$
\begin{aligned}
L & =E_{k}-E_{p} \\
& =\left(E_{k b}+E_{k a}\right)-\left(E_{p r}+E_{p a}\right)
\end{aligned}
$$

and the Lagrange's equation of motion

$$
\frac{\mathrm{d}}{\mathrm{d} t}\left[\frac{\partial L}{\partial \dot{\mathbf{q}}}\right]-\frac{\partial L}{\partial \mathbf{q}}+\frac{\partial S}{\partial \dot{\mathbf{q}}}=\boldsymbol{F}
$$

are used, where $\mathbf{q}$ is the displacements vector (which contains 3 translational and 3 rotational displacements of the rigid main body, and the displacements of flexible substructures), $\boldsymbol{F}$ is a general external forces vector, and $S$ is the Rayleigh's dissipation. The Rayleigh's dissipation is defined as

$$
S=\frac{1}{2} \dot{\mathbf{q}}^{T} \mathbf{D} \dot{\mathbf{q}}
$$

where $\mathbf{D}$ is a system damping matrix.

Then, it is assumed that control (external) forces acting on the rigid main body of the spacecraft are much larger than the forces resulting from the potential energy of the undeformed state. By using Eqs (5), (13), (27), (29), (34), (35), and (36), the equations of motion of the spacecraft then can be written as follows:

$$
\begin{aligned}
& m \ddot{\mathbf{r}}+\mathbf{Q} \dot{\boldsymbol{\omega}}+\mathbf{W} \ddot{\mathbf{d}}=\boldsymbol{F}_{b}-\boldsymbol{F}_{b}^{*}(\dot{\mathbf{r}}, \boldsymbol{\omega}, \dot{\mathbf{d}}), \\
& \mathbf{Q}^{T} \ddot{\mathbf{r}}+\mathbf{I} \dot{\boldsymbol{\omega}}+\mathbf{A} \ddot{\mathbf{d}}=\boldsymbol{T}_{b}-\boldsymbol{T}_{b}^{*}(\dot{\mathbf{r}}, \boldsymbol{\omega}, \dot{\mathbf{d}}) \\
& \mathbf{W}^{T} \ddot{\mathbf{r}}+\mathbf{A}^{T} \dot{\boldsymbol{\omega}}+\mathbf{M} \ddot{\mathbf{d}}+\mathbf{D} \dot{\mathbf{d}}+\mathbf{K d} \mathbf{d} \\
& =\boldsymbol{F}_{a}-\boldsymbol{F}_{a}^{*}(\dot{\mathbf{r}}, \boldsymbol{\omega}, \dot{\mathbf{d}})
\end{aligned}
$$

where $\mathbf{r}$ and $\boldsymbol{\omega}$ replace the notations of $\mathbf{r}_{i, b}$ and $\boldsymbol{\omega}_{b, i}$, $\boldsymbol{F}_{b}$ and $\boldsymbol{T}_{b}$ are external forces and torques vectors acting on the rigid main body, $\boldsymbol{F}_{a}$ has been explained in Eq. (31), and

$$
\boldsymbol{F}_{b}^{*}(\dot{\mathbf{r}}, \boldsymbol{\omega}, \dot{\mathbf{d}})=\tilde{\boldsymbol{\omega}}(m \dot{\mathbf{r}}+\mathbf{Q} \boldsymbol{\omega}+\mathbf{W} \dot{\mathbf{d}}),
$$

$$
\begin{aligned}
\boldsymbol{T}_{b}^{*}(\dot{\mathbf{r}}, \boldsymbol{\omega}, \dot{\mathbf{d}})= & \tilde{\boldsymbol{\omega}}\left(\mathbf{Q}^{T} \dot{\mathbf{r}}+\mathbf{I} \boldsymbol{\omega}+\mathbf{A} \dot{\mathbf{d}}\right) \\
& +\tilde{\mathbf{r}}(m \dot{\mathbf{r}}+\mathbf{Q} \boldsymbol{\omega}+\mathbf{W} \dot{\mathbf{d}}), \\
\boldsymbol{F}_{a}^{*}(\dot{\mathbf{r}}, \boldsymbol{\omega}, \dot{\mathbf{d}})= & \mathbf{B}(\dot{\mathbf{r}}, \boldsymbol{\omega}, \dot{\mathbf{d}}) \\
& \times\left(\mathbf{W}^{T} \dot{\mathbf{r}}+\mathbf{A}^{T} \boldsymbol{\omega}+\mathbf{M} \dot{\mathbf{d}}\right)
\end{aligned}
$$

are nonlinear terms containing multiplications between components of $\dot{\mathbf{r}}, \boldsymbol{\omega}$, and $\dot{\mathbf{d}}$, while $\mathbf{B}(\dot{\mathbf{r}}, \boldsymbol{\omega}, \dot{\mathbf{d}})$ in Eq. (42) is the matrix with elements consisting of linear combinations between components of $\dot{\mathbf{r}}, \boldsymbol{\omega}$, and $\dot{\mathbf{d}}$. In a lot of cases in attitude dynamics observation of spacecraft, values of the above nonlinear terms are small compared to the linear terms and can be neglected. This will result in terms of the linearized equations of motion of the flexible spacecraft. The linearized equations of motion will be used in the numerical simulations of this paper. For small Euler angles, they can be written in the matrix form as follows:

$$
\begin{aligned}
& {\left[\begin{array}{ccc}
m \mathbf{U}_{3} & \mathbf{Q} & \mathbf{W} \\
\mathbf{Q}^{T} & \mathbf{I} & \mathbf{A} \\
\mathbf{W}^{T} & \mathbf{A}^{T} & \mathbf{M}
\end{array}\right]\left\{\begin{array}{c}
\ddot{\mathbf{r}} \\
\ddot{\boldsymbol{\Theta}} \\
\ddot{\mathbf{d}}
\end{array}\right\}}
\end{aligned}
$$

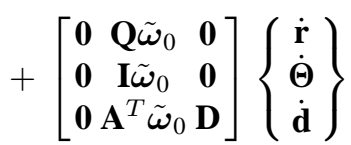

$$
\begin{aligned}
& +\left[\begin{array}{lll}
0 & 0 & 0 \\
0 & 0 & 0 \\
0 & 0 & \mathbf{K}
\end{array}\right]\left\{\begin{array}{l}
\mathbf{r} \\
\boldsymbol{\Theta} \\
\mathbf{d}
\end{array}\right\}=\left\{\begin{array}{l}
\boldsymbol{F}_{b} \\
\boldsymbol{T}_{b} \\
\boldsymbol{F}_{a}
\end{array}\right\},
\end{aligned}
$$

where $\tilde{\boldsymbol{\omega}}_{0}$ is the skew symmetric matrix of angular velocity of the satellite orbit $\boldsymbol{\omega}_{0}$, and $\Theta$ is a vector of Euler angles.

If the damping matrix $\mathbf{D}$ is supposed to be proportional damping, then $\mathbf{D}$ can be formed by linear combination of system mass matrix $\mathbf{M}$ and stiffness matrix K [16], i.e.,

$$
\mathbf{D}=\alpha \mathbf{M}+\beta \mathbf{K},
$$

where $\alpha$ and $\beta$ are constants. In this paper, numerical simulations will be carried out by supposing that the flexible structural subsystems have no dissipation properties, so, the values of $\alpha$ and $\beta$ in the above equation are equal to zero.

\subsection{Particular dynamical model of the satellite with solar panels being modeled as rectangular plate elements}

The mathematical expression of general spacecraft dynamics has been formed in Eqs (37-39). In this sub- 
section, a particular model of flexible spacecraft - a satellite consisting of a rigid main body carrying two symmetrical flexible solar panels - will be developed. To use Eqs (37-39), the mass matrix and the stiffness matrix need to be evaluated including flexible substructures. In this study, the attitude of the hypothetical satellite being chosen is as follows: the $Z_{b}$-axis of the rigid main body reference frame should point to the centre of earth, the $Y_{b}$-axis is normal to the orbital plane, and the $X_{b}$-axis should point to the velocity when there are no attitude errors.

For the application of finite element method to discretize elastic deformations of solar panels, the following idealizations are used:

(a) The solar panels are divided into rectangular flat plate bending elements.

(b) Each element has a uniform mass density.

(c) Only out-of-plane deformations of solar panels are considered.

(d) External loads (both forces and torques) on the solar panels are assumed to work on the nodal points of the elements.

(e) The $Y_{l}$-axes of elements and the $Y_{b}$-axis of main body frame are parallel or antiparallel (see Fig. 3). The $X_{l}$-axes and $Y_{l}$-axes of elements are in the panel plane, and their $Z_{l}$-axes are normal to the plane.

By using the above idealizations, each element of solar panel has 12 degrees of freedom in total, as shown by Fig. 5 .

In this research, the material of solar panels are assumed to be isotropic material. For an isotropic plate, $\mathbf{R}_{j}$ can be written as

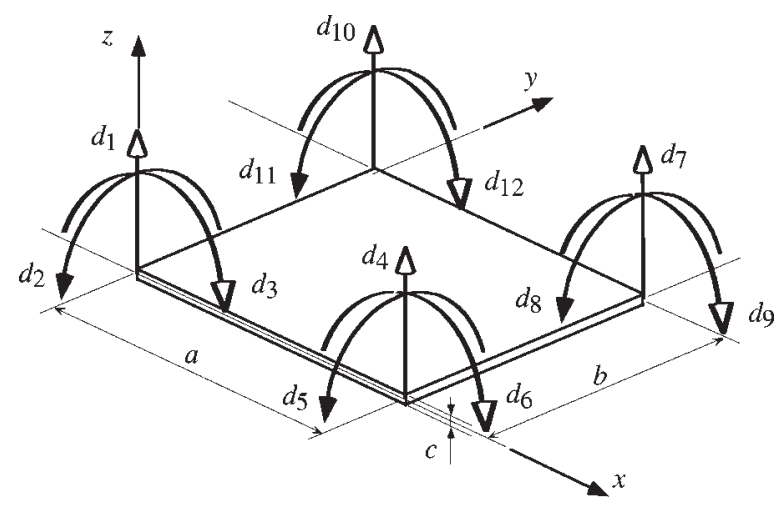

Fig. 5. A rectangular plate bending element model of solar panel.

$$
\mathbf{R}_{j}=\frac{E c^{3}}{12\left(1-\nu^{2}\right)}\left[\begin{array}{rrr}
1 & \nu & 0 \\
\nu & 1 & 0 \\
0 & 0 & \frac{1-\nu}{2}
\end{array}\right],
$$

where $E, c$ (see Fig. 5), and $\nu$ are the Young's modulus, thickness, and Poisson's ratio of $j$-th element respectively.

The shape function matrix introduced by Bogner [1] is selected. This shape function matrix can guarantee that deflections and slopes are all continuous on the edge of the element, and its expression for the $j$-th element is as follows:

$$
\mathbf{C}_{j}^{T}=\left[\begin{array}{c}
(1+2 \xi)(1-\xi)^{2}(1+2 \eta)(1-\eta)^{2} \\
(1+2 \xi)(1-\xi)^{2} \eta(1-\eta)^{2} b \\
-\xi(1-\xi)^{2}(1+2 \eta)(1-\eta)^{2} a \\
(1+2 \xi)(1-\xi)^{2}(3-2 \eta) \eta^{2} \\
-(1+2 \xi)(1-\xi)^{2}(1-\eta) \eta^{2} b \\
-\xi(1-\xi)^{2}(3-2 \eta) \eta^{2} a \\
(3-2 \xi) \xi^{2}(3-2 \eta) \eta^{2} \\
-(3-2 \xi) \xi^{2}(1-\eta) \eta^{2} b \\
(1-\xi) \xi^{2}(3-2 \eta) \eta^{2} a \\
(3-2 \xi) \xi^{2}(1+2 \eta)(1-\eta)^{2} \\
(3-2 \xi) \xi^{2} \eta(1-\eta)^{2} b \\
(1-\xi) \xi^{2}(1+2 \eta)(1-\eta)^{2} a
\end{array}\right]
$$

where $a$ and $b$ are the length and width of element respectively, $0 \leqslant\left(\xi=\frac{x}{a}\right) \leqslant 1$, and $0 \leqslant\left(\eta=\frac{y}{b}\right) \leqslant 1$ (see Fig. 5). For this shape function matrix, the coupling matrix $\mathbf{A}_{j}$ can be obtained as follows:

$$
\mathbf{A}_{j}^{T}=\frac{\rho a b c}{24}\left[\begin{array}{ccc}
6 y_{0_{j}}+\frac{9}{5} b & -6 x_{0_{j}}-\frac{9}{5} a & 0 \\
b y_{0_{j}}+\frac{2}{5} b^{2} & -b x_{0_{j}}-\frac{3}{10} a b & 0 \\
-a y_{0_{j}}-\frac{3}{10} a b & a x_{0_{j}}+\frac{2}{5} a^{2} & 0 \\
6 y_{0_{j}}+\frac{21}{5} b & -6 x_{0_{j}}-\frac{9}{5} a & 0 \\
-b y_{0_{j}}-\frac{3}{5} b^{2} & b x_{0_{j}}+\frac{3}{10} a b & 0 \\
-a y_{0_{j}}-\frac{7}{10} a b & a x_{0_{j}}+\frac{2}{5} a^{2} & 0 \\
6 y_{0_{j}}+\frac{21}{5} b & -6 x_{0_{j}}-\frac{21}{5} a & 0 \\
-b y_{0_{j}}-\frac{3}{5} b^{2} & b x_{0_{j}}+\frac{7}{10} a b & 0 \\
a y_{0_{j}}+\frac{7}{10} a b & -a x_{0_{j}}-\frac{3}{5} a^{2} & 0 \\
6 y_{0_{j}}+\frac{9}{5} b & -6 x_{0_{j}}-\frac{21}{5} a & 0 \\
b y_{0_{j}}+\frac{2}{5} b^{2} & -b x_{0_{j}}-\frac{7}{10} a b & 0 \\
a y_{0_{j}}+\frac{3}{10} a b & -a x_{0_{j}}-\frac{3}{5} a^{2} & 0
\end{array}\right],
$$

where $x_{0_{j}}$ and $y_{0_{j}}$ are the components of ${ }^{l} \mathbf{r}_{b, o}$ (see Fig. 3) for the $j$-th element along its $X_{l}$ and $Y_{l}$-axes re- 
spectively, $\rho$ is the mass density, while the inertia matrix $\mathbf{I}_{j}$ can be written as follows:

$$
\mathbf{I}_{j}=\rho a b c\left[\begin{array}{lll}
I_{j_{11}} & I_{j_{21}} & I_{j_{31}} \\
I_{j_{21}} & I_{j_{22}} & I_{j_{32}} \\
I_{j_{31}} & I_{j_{32}} & I_{j_{33}}
\end{array}\right]
$$

where

$$
\begin{aligned}
& I_{j_{11}}=y_{0_{j}}^{2}+\frac{1}{12} c^{2}+\frac{1}{3} b^{2}+y_{0_{j}} b, \\
& I_{j_{21}}=-\frac{1}{2}\left(\frac{1}{2} a b+y_{0_{j}} a+x_{0_{j}} b\right)-x_{0_{j}} y_{0_{j}}, \\
& I_{j_{31}}=I_{j_{32}}=0, \\
& I_{j_{22}}=x_{0_{j}}^{2}+\frac{1}{12} c^{2}+\frac{1}{3} a^{2}+x_{0_{j}} a, \\
& I_{j_{33}}=x_{0_{j}}^{2}+y_{0_{j}}^{2}+\frac{1}{3}\left(a^{2}+b^{2}\right)+x_{0_{j}} a+y_{0_{j}} b,
\end{aligned}
$$

and the coupling matrix for rotational displacements of the main body and the displacements of element $j$ can be written as follows:

$$
\mathbf{W}_{j}=\frac{\rho a b c}{24}\left[\begin{array}{cccccccccccc}
0 & 0 & 0 & 0 & 0 & 0 & 0 & 0 & 0 & 0 & 0 & 0 \\
0 & 0 & 0 & 0 & 0 & 0 & 0 & 0 & 0 & 0 & 0 & 0 \\
6 & b & -a & 6 & -b & -a & 6 & -b & a & 6 & b & a
\end{array}\right] .
$$

\section{Rest-to-rest 3-dimensional attitude maneuver of flexible satellite under bang-bang roll torque input}

If time is the independent variable and the initial values of dependent variables are given in addition to boundary conditions, the dynamics of the satellite can be simulated numerically. A lot of methods can be used to solve the set of differential equations of motion, Eqs (43). In this simulations, the unconditionally stable of Newmark method is selected.

In this study, the observed satellite has no control and no damping properties on the flexible solar pan-

Table 1

Lumped masses consisting of the rigid main body.

\begin{tabular}{crrr}
\hline \multirow{2}{*}{$\begin{array}{c}\text { Mass } \\
\mathrm{kg})\end{array}$} & $x_{b}$ & $y_{b}$ & $z_{b}$ \\
\hline & 0.40 & 0.00 & 0.00 \\
400 & -0.40 & 0.00 & 0.00 \\
400 & 0.00 & 0.50 & 0.00 \\
500 & 0.00 & -0.50 & 0.00 \\
500 & 0.00 & 0.00 & 1.40 \\
550 & 0.00 & 0.00 & -1.40 \\
550 & & & \\
\hline
\end{tabular}

els. The control inputs are only applied to the rigid main body at the center of mass of satellite, as on-off reaction jets with constant amplitude force or torque pulses. For such a system, under control or external torques only, remembering Eqs (43), the resultant attitude angle acceleration of satellite as a rigid body motion can be written as

$$
\left\{\begin{array}{c}
\ddot{\phi} \\
\ddot{\theta} \\
\ddot{\psi}
\end{array}\right\}=\left[\begin{array}{lll}
I_{x x} & I_{x y} & I_{x z} \\
I_{x y} & I_{y y} & I_{y z} \\
I_{x z} & I_{y z} & I_{z z}
\end{array}\right]^{-1}\left\{\begin{array}{l}
T_{b_{x}}(t) \\
T_{b_{y}}(t) \\
T_{b_{z}}(t)
\end{array}\right\},
$$

where $I_{x x}, I_{y y}, I_{z z}, I_{x y}, I_{x z}$, and $I_{y z}$ are components of the inertia matrix $\mathbf{I}$ of the whole satellite, and $T_{b_{x}}$, $T_{b_{y}}$, and $T_{b_{z}}$ are components of the torque input vector $\mathbf{T}_{b}$ on the rigid main body. By integrating Eq. (50) with respect to time we get an expression of desired attitude angle velocity,

$$
\left\{\begin{array}{c}
\dot{\phi}_{d} \\
\dot{\theta}_{d} \\
\dot{\psi}_{d}
\end{array}\right\}=\int\left[\begin{array}{lll}
I_{x x} & I_{x y} & I_{x z} \\
I_{x y} & I_{y y} & I_{y z} \\
I_{x z} & I_{y z} & I_{z z}
\end{array}\right]^{-1}\left\{\begin{array}{l}
T_{b_{x}}(t) \\
T_{b_{y}}(t) \\
T_{b_{z}}(t)
\end{array}\right\} \mathrm{d} t
$$

and integrating once more gives a desired roll angle displacement,

$$
\left\{\begin{array}{c}
\phi_{d} \\
\theta_{d} \\
\psi_{d}
\end{array}\right\}=\iint\left[\begin{array}{lll}
I_{x x} & I_{x y} & I_{x z} \\
I_{x y} & I_{y y} & I_{y z} \\
I_{x z} & I_{y z} & I_{z z}
\end{array}\right]^{-1}\left\{\begin{array}{l}
T_{b_{x}}(t) \\
T_{b_{y}}(t) \\
T_{b_{z}}(t)
\end{array}\right\} \mathrm{d} t \mathrm{~d} t .
$$

The main body of satellite is modeled as 6 lumped masses at certain positions as be shown in Table 1, and the parameters of flexible solar panels can be seen in Table 2. The offset angle of solar panels is taken to be 30 degrees. For this configuration, the origin

Table 2

Parameters of the solar panels of satellite

\begin{tabular}{ll}
\hline \multicolumn{1}{c}{ Description } & \multicolumn{1}{c}{ Values } \\
\hline Number of solar panels & 2 \\
Dimension of each solar panel $\left(\mathrm{m}^{3}\right)$ & $12 \times 2.4 \times 0.03$ \\
Young's modulus, $E\left(\mathrm{~N} / \mathrm{m}^{2}\right)$ & $0.6 \times 10^{8}$ \\
Poisson ratio, $\nu$ & 0.3 \\
Mass density, $\rho\left(\mathrm{kg} / \mathrm{m}^{3}\right)$ & 120 \\
Number of elements in each solar panel & 16 \\
Dimension of each element, $b \times a \times c\left(\mathrm{~m}^{3}\right)$ & $1.5 \times 1.2 \times 0.03$ \\
Offset angle, $\delta($ degrees $)$ & 30 \\
Damping coefficients, $(\alpha, \beta)$ & $(0,0)$ \\
Distance between panel's root and $O_{b}(\mathrm{~m})$ & 1.80 \\
\hline
\end{tabular}




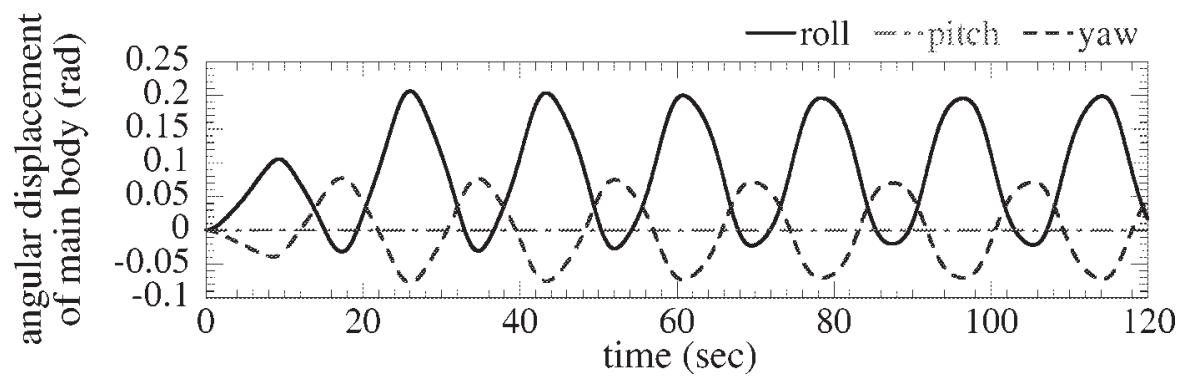

(a)

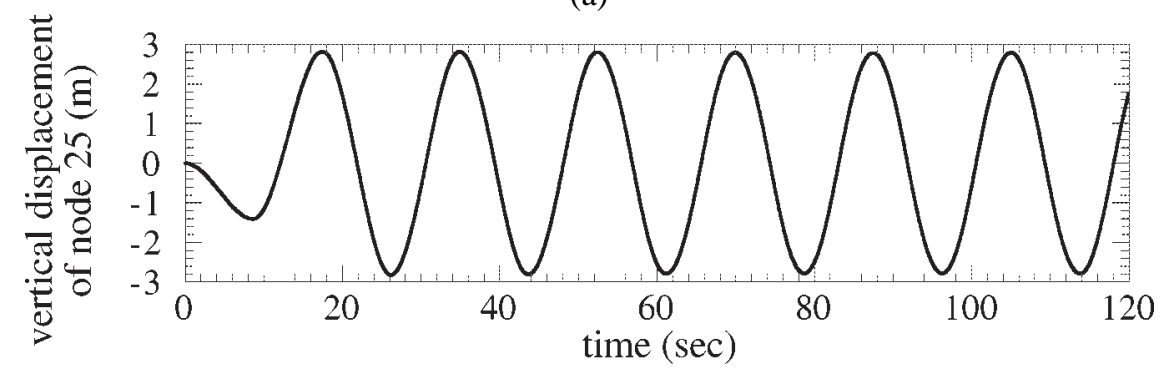

(b)

Fig. 6. Time responses under the bang-bang roll and yaw torque inputs: (a) attitude angle displacement of the main body, (b) vertical displacement measured in local reference frame of node 25 of solar panel.

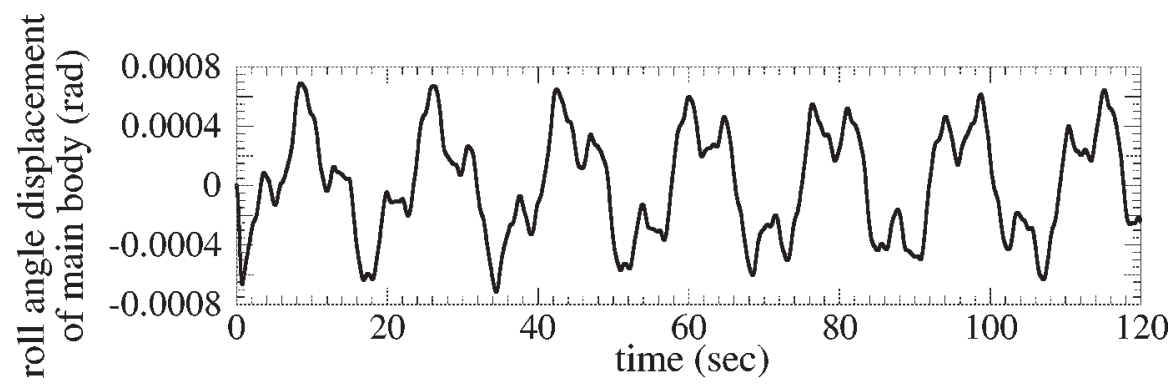

(a)

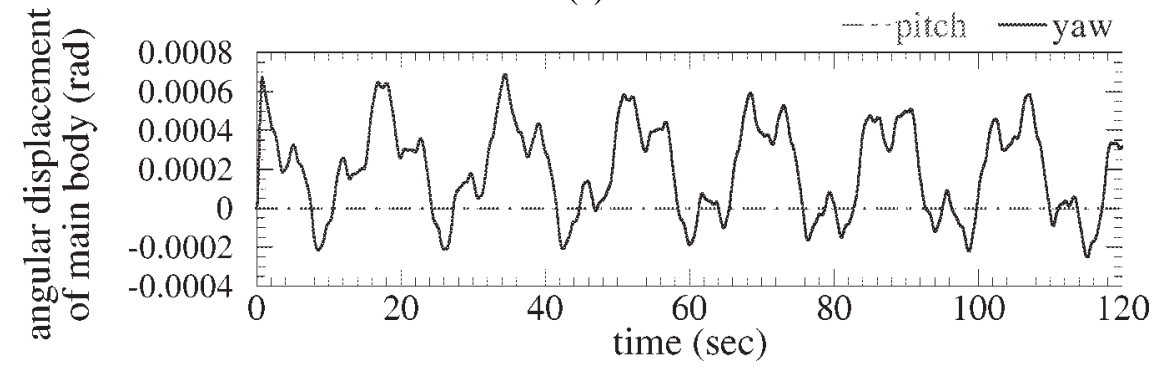

(b)

Fig. 7. Time responses of the rigid main body under the bang-bang yaw torque input only: (a) roll angle displacement, (b) pitch and yaw angle displacements. 
of the rigid main body fixed reference frame coincides with the centre of mass of the whole satellite in the undeformed state, $I_{x x}=17,535 \mathrm{~kg} \mathrm{~m}^{2}, I_{y y}=$ $2,384 \mathrm{~kg} \mathrm{~m}^{2}, I_{z z}=15,557 \mathrm{~kg} \mathrm{~m}^{2}, I_{x y}=I_{y z}=0$, and $I_{x z}=43 \mathrm{~kg} \mathrm{~m}^{2}$. The initial condition of the satellite is given to be the undeformed state; the main body fixed frame and the orbital reference frame coincide with the inertial reference frame. The orbital frame moves relative to the inertial frame with constant angular velocity

$$
\boldsymbol{\omega}_{o}=-\omega_{o} \boldsymbol{j}_{i}
$$

where $\boldsymbol{j}_{i}$ is the unit vector in $Y_{i}$-axis direction, $\omega_{o}=$ $7.29 \times 10^{-5} \mathrm{rad} / \mathrm{s}$, so that $F_{o}$ performs in $F_{i}$ one rotation per sidereal day ( 24 hours of sidereal time or 23 hours 56 minutes 4.09054 seconds of mean solar time). The roll angle of the satellite will be changed to the desired angle of $5^{\circ}$, or about $0.0873 \mathrm{rad}$, while the pitch and yaw angles are still $0^{\circ}$. The shortest duration time of constant-amplitude command for restto-rest slew maneuver is a bang-bang input. Constraint equations that must be satisfied for this rest-to-rest slew maneuver are $\left\{\dot{\phi}_{d}, \dot{\theta}_{d}, \dot{\psi}_{d}\right\}^{T}=\{0,0,0\}^{T}$ and $\left\{\phi_{d}, \theta_{d}, \psi_{d}\right\}^{T}=\{0.0873,0,0\}^{T}$. If the amplitude of command, either $T_{b_{x}}, T_{b_{y}}$, or $T_{b_{z}}$ is determined to be $20 \mathrm{Nm}$, the profile of bang-bang torques needed consist of 17.494 seconds long of $T_{b_{x}}$ and $0.868 \mathrm{sec}-$ onds long of $T_{b_{z}}$ bang-bangs. The satellite is subjected firstly to the roll torque input, then after the roll input was removed, the yaw roll input is given directly. Under these inputs, the roll angle attitude will change, yaw angle is disturbed, while pitch angle is not excited. The roll angle changes to the desired displacement, but after roll maneuver input was stopped, the roll angle still oscillates with a very large amplitude dominantly at the period of 17.49 seconds, which relates to natural frequency resulted in calculation $0.3593 \mathrm{rad} / \mathrm{s}$. The total amplitude of residual oscillation reaches until $0.118 \mathrm{rad}$ (or $6.76^{\circ}$ ) for roll angle, as shown in Fig. 6(a), and $0.078 \mathrm{rad}$ (or $4.47^{\circ}$ ) for yaw angle. This residual roll angle oscillation is about $135 \%$ of the desired roll displacement. Of course such residual oscillation is very unacceptable and disturbs the mission of the satellite. The largest residual vibration on solar panels take place at their tips. The node 25 , shown in Fig. 6(b), experiences unlikely local vertical vibration with high deflection amplitude of 2.847 meters. Compared with solar panel's length of $12 \mathrm{~m}$, this deflection is about $24 \%$, a condition where the use of local reference frame for its element in finite element method fixed at its initial condition is no longer true.
The influence of the bang-bang yaw input needed to increase or decrease residual attitude angle oscillations of the satellite is very small. In the simulation, when the satellite is subjected to this yaw input only, the rest condition of roll angle moves to $-0.000005 \mathrm{rad}$ (or $-0.001^{\circ}$ ) with the residual roll angle oscillation in total amplitude of less then $0.00071 \mathrm{rad}$ (or $0.04^{\circ}$ ), as shown in Fig. 7(a). The pitch angle is undisturbed, its value is still zero. The rest of yaw angle changes to $0.00022 \mathrm{rad}$ (or $0.01^{\circ}$ ) with residual yaw angle oscillation in total amplitude of less than $0.00047 \mathrm{rad}$ (or $0.03^{\circ}$ ). The time responses of pitch and yaw angles can be seen in Fig. 7(b).

\section{Input shaper to reduce residual vibration in rest-to-rest slew maneuvers}

The flexible satellite studied here is equipped with on-off reaction jets, so it cannot produce variable amplitude actuation force; the satellite must be moved with constant amplitude force pulses. For this kind of satellite, when the bang-bang input was used for restto-rest slew maneuvers, the residual vibration of flexible members and attitude oscillation of rigid main body after slewing maneuver input was removed can be relatively large enough compared with the desired new attitude. By shaping the input, the residual vibration can be reduced.

The inputs to the system under consideration must consist only of positive or negative constant-amplitude force pulses. For rest-to-rest slews, the commands must contain both positive and negative pulses so that the system can be accelerated and then decelerated back to zero velocity. A series of alternating-sign pulses for rest-to-rest time optimal slew maneuver can be generated by convolving a step with an input shaper of the form [14]

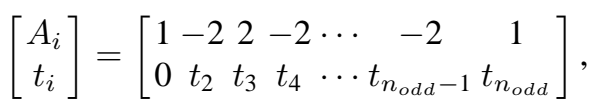

where $i=1, \ldots, n_{\text {odd }}, A_{i}$ is the amplitude of the $i$-th impulse, $t_{i}$ is the time location of the $i$-th impulse, and $n_{\text {odd }}$ is odd.

Figure 8 demonstrates Eq. (54), that a step convolved with this type of an input shaper results in a series of alternating sign, variable-width pulses. Eq. (54) leads to our first constraint equations for rest-to-rest shapers: 


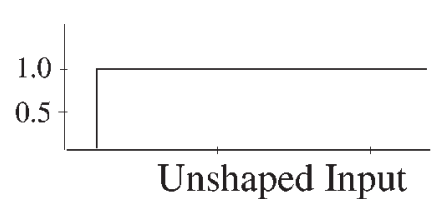

*
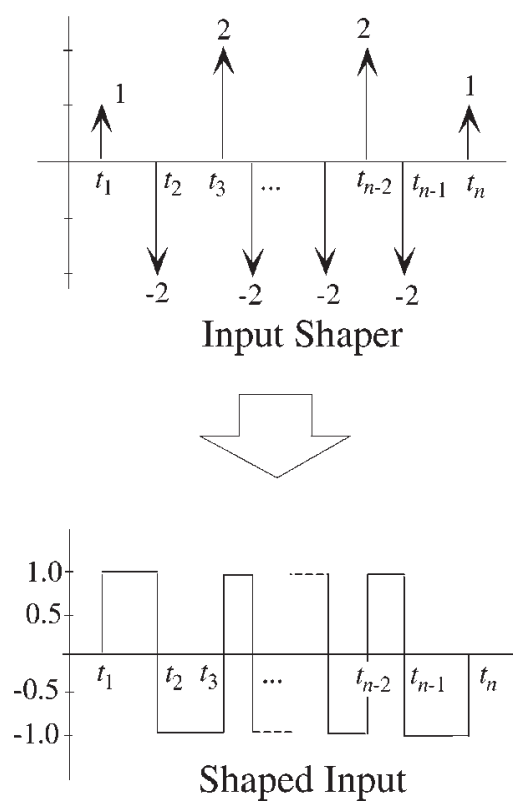

Fig. 8. Input shaping to generate a series of alternating-sign pulses.

$$
\begin{cases}A_{i}=1, & i=1, n_{\text {odd }}, \\ A_{i}=2(-1)^{(i-1)}, & i=2,3, \ldots,\left(n_{\text {odd }}-1\right) .\end{cases}
$$

The next constraint equations must ensure that the system's mass centre will move the desired amount. For the system under torque inputs as studied in this paper, the equations (51) and (52) of the previous section are for the attitude angle maneuvers of rigid-body motion; however, the main purpose of the input shaping is to limit the amount of residual vibration that occurs when the system reaches its desired setting point. The constraint equation limiting vibration amplitude can be formulated as a ratio of residual vibration amplitude with input shaping divided by residual vibration without shaping. When the techniques are designed for use with on-off actuators, it would be appropriate to use a baseline input. Whenever possible, the bang-bang input is used as the standard for commands that slew from rest to rest.

A bang-bang input can be viewed as a step input convolved with a shaper of the form

$$
\left[\begin{array}{c}
A_{i} \\
t_{i}
\end{array}\right]=\left[\begin{array}{ccc}
1 & -2 & 1 \\
0 & t_{b b_{s}} & t_{b b_{l}}
\end{array}\right]
$$

where $t_{b b_{s}}$ is the switching time and $t_{b b_{l}}$ is the length of the bang-bang. This is demonstrated by the first three impulses in Fig. 8 if the value of the third impulse is changed from 2 to 1 .

The amplitude of residual vibration of an undamped second-order system when subjected to a sequence of impulses can be expressed as a summation of the responses to individual impulses that is given in the reference [2]. The expression for the amplitude is

$$
\begin{aligned}
& R_{a m p}(\omega)= \\
& \quad \sqrt{\left[\sum A_{i} \sin \left(\omega t_{i}\right)\right]^{2}+\left[\sum A_{i} \cos \left(\omega t_{i}\right)\right]^{2}},
\end{aligned}
$$

where $\omega$ is the undamped natural frequency. We can construct the ratio of shaped to unshaped vibration by dividing Eq. (55) for the input shaper by the equivalent Eq. (57) for the impulse sequence corresponding to the unshaped input. The percentage vibration relative to a bang-bang is

$V(\omega)=$

$$
\sqrt{\frac{\left[\sum A_{i} \sin \left(\omega t_{i}\right)\right]^{2}+\left[\sum A_{i} \cos \left(\omega t_{i}\right)\right]^{2}}{\left[\sum A_{b b_{j}} \sin \left(\omega t_{b b_{j}}\right)\right]^{2}+\left[\sum A_{b b_{j}} \cos \left(\omega t_{b b_{j}}\right)\right]^{2}}},
$$

where $A_{b b_{j}}$ and $t_{b b_{j}}$ describe the input shaper corresponding to the bang-bang and are given by Eq. (56).

By including Eq. (58) in our set of constraint equations, we can set the level of vibration at the system's frequency $\omega$ to the quantity $V$. For example, when $V(\omega)=0.05$, the shaped vibration amplitude at the frequency $\omega$ is $5 \%$ of the baseline vibration amplitude at the same frequency. The known zero vibration (ZV) shaper uses the criteria of $V=0$. For systems containing modelling errors, several kinds of input shaper have been proposed by researchers, for example zero vibration and derivative (ZVD) shaper [13] and extrainsensitive (EI) shaper [14]. However, the range of frequencies of satellite studied in this paper is very wide. It varies from $1.078 \times 10^{-15} \mathrm{rad} / \mathrm{s}$ (or $1.717 \times 10^{-16} \mathrm{~Hz}$ ) up to $343.4 \mathrm{rad} / \mathrm{s}$ (or $54.65 \mathrm{~Hz}$ ), and there may exist a large amount of residual vibration.

\section{Rest-to-rest attitude maneuver under shaped inputs}

The attitude maneuvers of the flexible satellite under shaped torque inputs are observed in this section in 


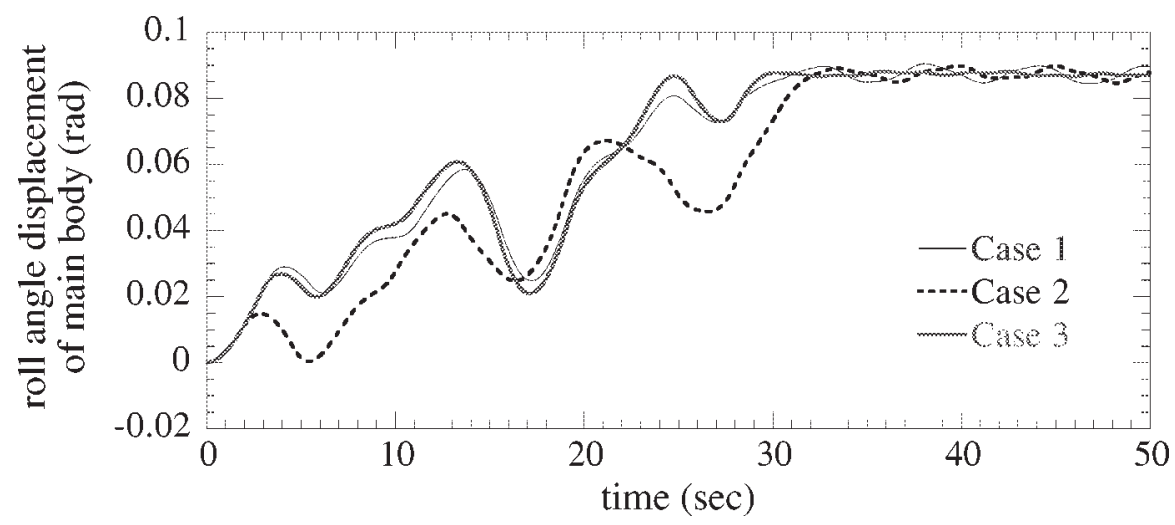

(a)

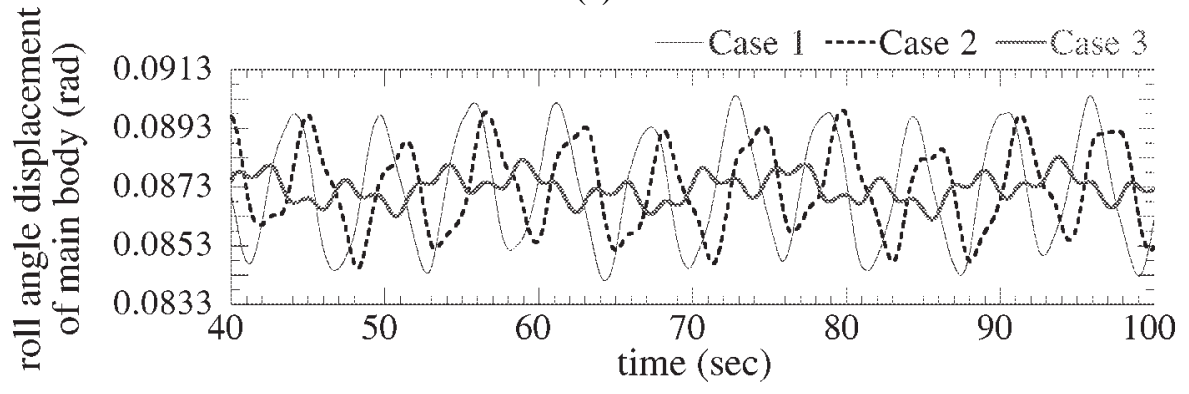

(b)

Fig. 9. Time responses of roll angle displacement of the main body: (a) during maneuver, (b) residual oscillation.

Table 3

Time location of impulses for shaping the roll torque inputs

\begin{tabular}{lccccccccccc}
\hline Case & $t_{1}$ & $t_{2}$ & $t_{3}$ & $t_{4}$ & \multicolumn{1}{c}{$t_{5}$} & \multicolumn{1}{c}{$t_{6}$} & $t_{7}$ & $t_{8}$ & $t_{9}$ & $V(0.9563)$ & $V(1.1166)$ \\
number & $(\mathrm{s})$ & $(\mathrm{s})$ & \multicolumn{1}{c}{$(\mathrm{s})$} & \multicolumn{1}{c}{$(\mathrm{s})$} & \multicolumn{1}{c}{$(\mathrm{s})$} & \multicolumn{1}{c}{$(\mathrm{s})$} & \multicolumn{1}{c}{$(\mathrm{s})$} & \multicolumn{1}{c}{$(\mathrm{s})$} & \multicolumn{1}{c}{$(\mathrm{s})$} & \multicolumn{1}{c}{$(\%)$} & $(\%)$ \\
\hline Case 1 & 0 & 3.01 & 5.41 & 10.93 & 15.17 & 18.75 & 24.68 & 27.17 & 29.20 & 26.8 & 42.1 \\
Case 2 & 0 & 1.86 & 4.57 & 9.85 & 13.23 & 18.51 & 24.10 & 27.33 & 31.30 & 220.7 & 34.3 \\
Case 3 & 0 & 2.84 & 5.06 & 10.70 & 15.06 & 18.70 & 24.96 & 27.44 & 29.20 & 65.2 & 11.3 \\
\hline
\end{tabular}

order to change its roll angle to the desired value with residual oscillations of attitude angles not greater then permissible pointing errors of the satellite. For the system having a lot of flexible modes such as the finite element model of satellite studied here, when the vibration at a natural frequency with the strongest residual vibration is suppressed, for example to be zero, other frequencies can amplify the resulted residual vibration so that it is still relatively large enough to exceed the expected level for the mission. In such a case, the vibration at several natural frequencies needs to be reduced until the resultant residual vibration is within the acceptable level.

The desired maneuver of the satellite observed in this study is $5^{\circ}$ roll angle displacement, and the residual oscillations are determined to be not greater than $0.07^{\circ}$ in roll and pitch angles, and not greater than $0.2^{\circ}$ in yaw angle. These values of residual angular oscillations are such that they are equivalent to the permissible maximum antenna beam pointing errors of the satellite required by the KOREASAT [3]. When bangbang torque inputs are used for maneuver as described in the Section 3, see Fig. 6, the very strong residual roll and yaw oscillations happens at $\omega=0.3593 \mathrm{rad} / \mathrm{s}$, and these are caused by the roll input. The yaw bang-bang input is for a short duration and has relatively small enough contribution to the residual attitude oscillation (Fig. 7), so that, in order to satisfy the satellite mission, an input shaper is applied to the roll torque only. However, the bang-bang yaw input is still applied after the shaped roll input is switched off. Remembering that the permissible maximum amplitude of roll oscil- 


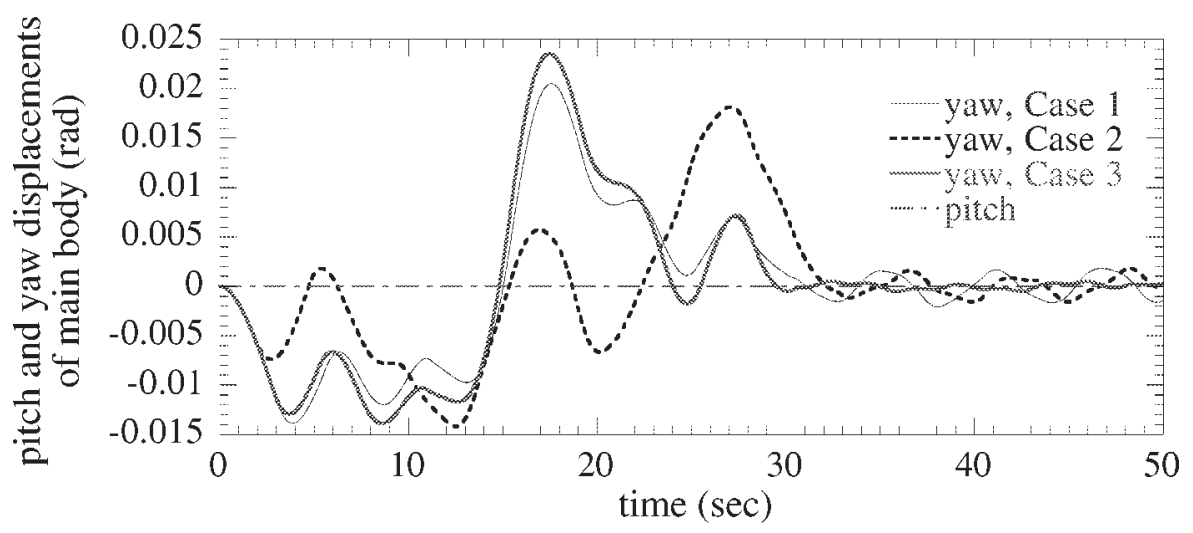

(a)

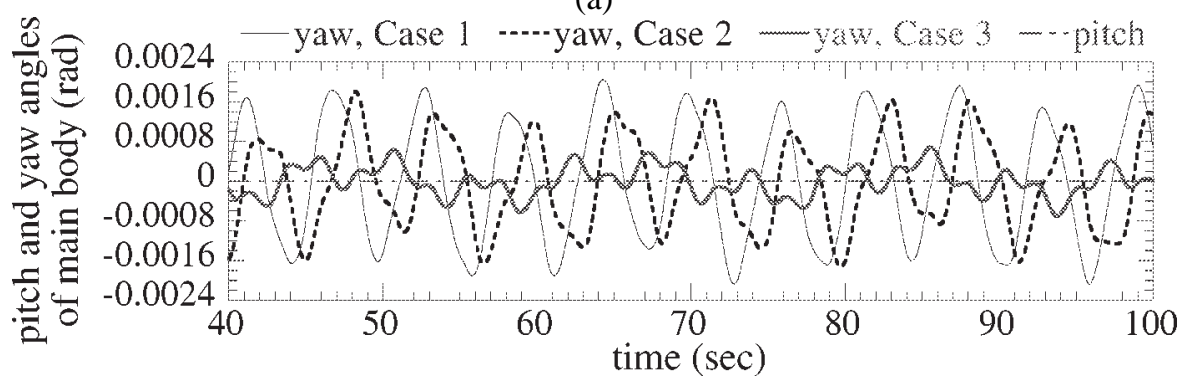

(b)

Fig. 10. Time responses of pitch and yaw angle displacements of the main body: (a) during maneuver, (b) residual oscillation.

lation is $0.07^{\circ}$, while its oscillation caused by the bangbang yaw input is about $0.04^{\circ}$, the resultant residual oscillation caused by the shaped input must be smaller than $0.03^{\circ}$ (or $0.4 \%$ of the result of bang-bang roll input). In order to reach this value, the roll torque input shaper with the same constant-amplitude of $20 \mathrm{Nm}$ is developed in this study by setting the percentage of vibration at $\omega=0.3593 \mathrm{rad} / \mathrm{s}, V(0.3593)$, to zero. Besides this natural frequency, the reduction of vibration at two higher natural frequencies of the system (i.e., $\omega=0.9563$ and $1.1166 \mathrm{rad} / \mathrm{s}$ ) is considered. The number of impulses is selected to be 9 in time duration of command less than 40 seconds. By using these constraints, $V(0.9563)$ and $V(1.1166)$ never be zero. The shaped input with the smallest value of $V(0.9563)$ is listed in Case 1 of Table 3, while the one with the smallest value of $V(1.1166)$ is written in Case 3. To know at which natural frequency the resultant vibration gives a larger response amplitude, the command with the relatively small $V(1.1166)$ but the very large $V(0.9563)$ is chosen as listed in Case 2.

Residual roll oscillation. The roll angle motions of the main rigid body of satellite for all cases are plotted in Fig. 9. We can see in Fig. 9(a), that the rest of roll angle has been changed successfully to the desired new value of $5^{\circ}$ (0.0873 rad) after attitude maneuvers. For Case 1, where the percentages of vibration are $26.8 \%$ at $\omega=0.9563 \mathrm{rad} / \mathrm{s}$ and $42.1 \%$ at $\omega=1.1166 \mathrm{rad} / \mathrm{s}$, the total amplitude of residual roll oscillation is about $0.0032 \mathrm{rad}\left(0.18^{\circ}\right)$, strongly at the period of $5.63 \mathrm{~s}$ ( or $\omega=0.9563 \mathrm{rad} / \mathrm{s}$ ). It means that the use of input of Case 1 reduces the total amplitude of residual roll angle oscillation at about $2.7 \%$ compared with the result of the bang-bang input, but it is still greater, about 2.6 times, than its permissible maximum error. In Case 2 percentages of vibration are $220.7 \%$ at $\omega=0.9563 \mathrm{rad} / \mathrm{s}$ and $34.3 \%$ at $\omega=1.1166 \mathrm{rad} / \mathrm{s}$ respectively and gives the total amplitude of residual roll angle oscillation to be about $0.0028 \mathrm{rad}(0.16$ degrees), the reduction of about $2.4 \%$ compared with the result of bang-bang input, but it is still greater than the permissible value. The very small residual roll angle oscillation after maneuver is given by Case 3 , where the percentages of vibration are $65.2 \%$ at $0.9563 \mathrm{rad} / \mathrm{s}$ and $11.3 \%$ at $1.1166 \mathrm{rad} / \mathrm{s}$. Its total amplitude is about $0.0011 \mathrm{rad}\left(\right.$ or $0.06^{\circ}$ ), or $0.9 \%$ of the result of bang-bang input. This value satisfies the precise pointing requirement of the satellite. 


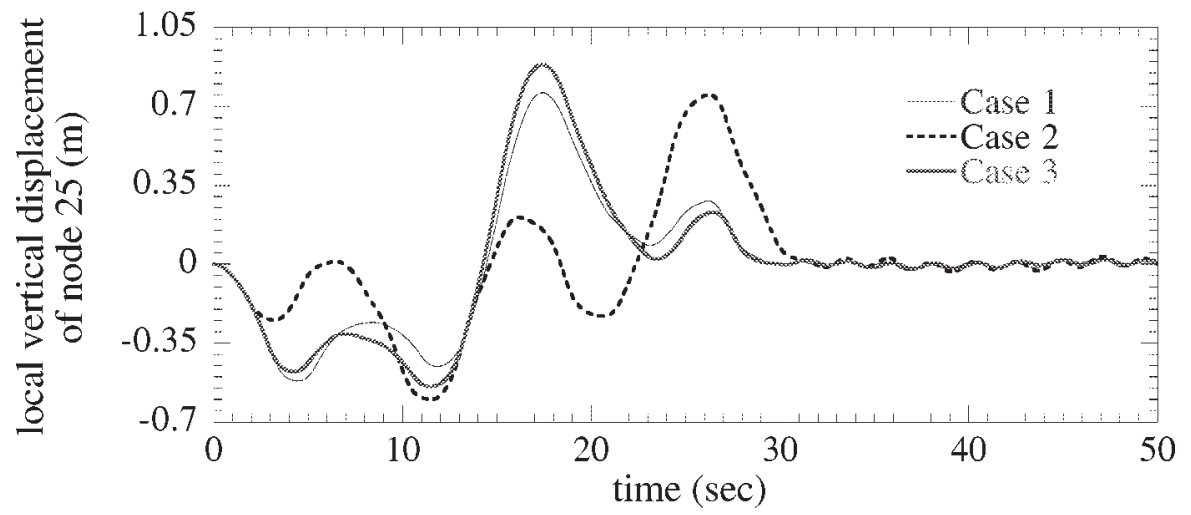

(a)

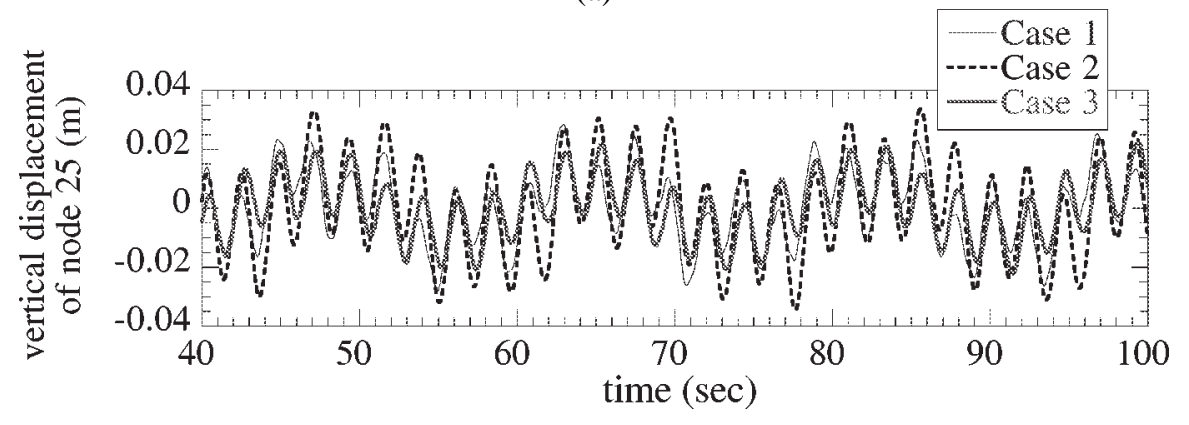

(b)

Fig. 11. Time responses of local vertical displacement of node 25 of solar panel: (a) during maneuver, (b) residual vibration.

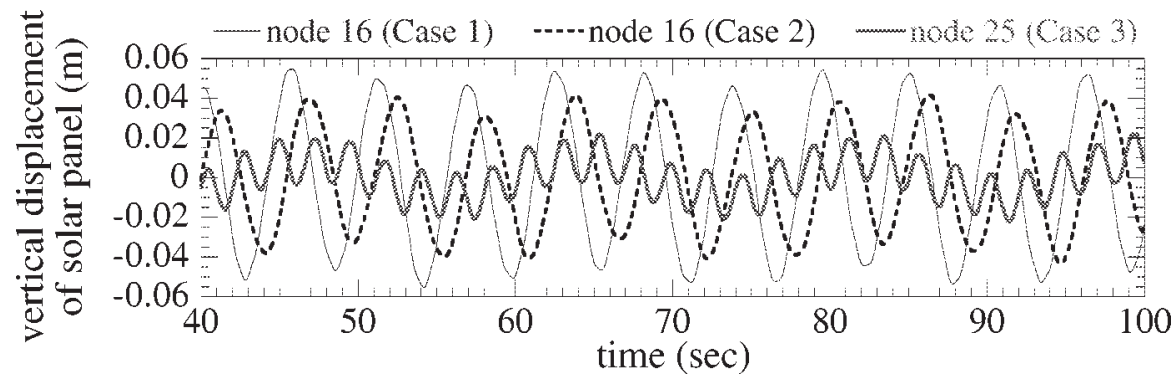

Fig. 12. Time responses of the largest residual vibration of solar panel for all cases.

Remembering the percentages of vibration at $\omega=$ 0.9563 and $1.1166 \mathrm{rad} / \mathrm{s}$ achieved by each case in the connection with their resultant residual roll oscillations, the model of satellite observed in this study has a tendency to give a larger response amplitude at 1.1166 $\mathrm{rad} / \mathrm{s}$ compared with the $0.9563 \mathrm{rad} / \mathrm{s}$ one. Besides its $0.3593 \mathrm{rad} / \mathrm{s}$ natural frequency, we must give attention to the value of percentage vibration at $1.1166 \mathrm{rad} / \mathrm{s}$ in selecting shaped inputs in order to reduce residual roll oscillation within the desired level.
Residual pitch and yaw oscillations. For all cases of switching time listed in Table 3, the pitch angles are undisturbed, as shown in Fig. 10, while the rest of yaw angle after maneuvers are still zero. Case 3 gives the largest maximum yaw angle motion during the attitude maneuver, about 0.0235 rad or $1.35^{\circ}$, but the smallest residual one after the inputs were removed. It can be seen in Fig. 10(b) that its total amplitude of residual yaw oscillation never exceeds $0.0008 \mathrm{rad}\left(0.05^{\circ}\right)$, while the total amplitudes of Cases 1 and 2 are smaller than $0.0022 \operatorname{rad}\left(0.17^{\circ}\right)$. These values satisfy the re- 
quirement of satellite in terms of the maximum yaw pointing error as previously discussed.

Maximum deflections of solar panels. Since the solar panels of satellite are supposed to have only outof-plane elastic deformations, the deflections of their nodes are symmetric with respect to $Y_{b}$ and antisymmetric with respect to $X_{b}$. It means that the deflection of node 25 at each time of observation is the same as that at node 27, and the deflections of nodes 52 and 54 have identical magnitudes but have opposite directions. The maximum deflections of solar panels happen at their tips, i.e., nodes $25,27,52$, and 54, and as a representation, in this paper, time responses of node 25 are given. Case 3 , where sequence impulsing time intervals of the input shaper at the range of 1.76 $\mathrm{s}$ until $6.26 \mathrm{~s}$ gives the largest maximum deflection of node 25 as seen in Fig. 11(a), about $88.6 \mathrm{~cm}$ upward at $t=17.41 \mathrm{~s}$. Compared with the length of solar panel, this deflection is only about $7.4 \%$. The Case 1 with intervals of sequence impulsing time from $2.03 \mathrm{~s}$ until $5.93 \mathrm{~s}$ gives the $76.4 \mathrm{~cm}$ maximum deflection of this node, and Case 2 with the intervals of 1.86 until $5.59 \mathrm{~s}$ gives the $75.5 \mathrm{~cm}$ maximum deflection. So, we can see that, the larger the value of the longest intervals of sequence impulsing time, the larger is the maximum deflection on solar panel. The total amplitudes of residual vibration of node 25 for all cases are smaller than $4 \mathrm{~cm}$, as shown in Fig. 11(b).

Maximum residual vibration of solar panels. The use of shaped inputs has reduced successfully the residual vibrations of flexible modes. The maximum residual vibrations of solar panels for Cases 1 and 2 happen at nodes 16, 18, 43, and 45; while for Case 3, it happens at the tips of panels, i.e., nodes $25,27,52$, and 54 . The total amplitudes of maximum residual vibration of panels for all cases are smaller than $5.5 \mathrm{~cm}$ (about $0.5 \%$ of solar panel's length), as shown in Fig. 12, where the smallest one happens in Case 3 at the value of $2.3 \mathrm{~cm}$.

\section{Concluding remarks}

Attitude maneuvers of the flexible satellite induce the vibration of flexible members as well as the satellite libration motion. However, for the satellite in a symmetrical condition with respect to $Y_{b}$-axis of rigid main body fixed frame, i.e., $I_{x y}$ and $I_{y z}$ equal to zeros, under the roll and yaw torque inputs, the pitch motions is not induced. Without shaping the input, the flexible satellite has poor attitude accuracy after slew ma- neuver. Under the combination of bang-bang roll and yaw torque input, for 5 degrees desired roll angle displacement only, the satellite studied in this paper has residual roll angle oscillation with amplitude greater than the slew maneuver angle. Shaped inputs show their capability to reduce residual oscillation and vibration slightly. For the shaped inputs, nine impulses are utilized here and the resultant maximum total amplitudes of residual roll oscillation become smaller than 0.18 degrees, the largest total amplitudes of residual vibration of 12 meters solar panel's length are not greater than $5.5 \mathrm{~cm}$, and the total amplitudes of tip solar panel's residual vibration are not greater than $4 \mathrm{~cm}$.

The selection of shaped input with zero value of percentage of vibration at $\omega$ of the strongest residual vibration and relatively small values at other strong ones will give the attitude maneuver with the small residual attitude angle oscillations satisfying the mission requirement of satellite in terms of pointing accuracy. To maneuver flexible spacecraft with small maximum deflection, the input shaper consisting of short intervals of sequence impulsing times is needed.

\section{References}

[1] F.K. Bogner, R.H. Mallet, M.D. Minick and L.A. Schmidt, Development and evaluation of energy searh methods of nonlinear structural analysis. Flight Dynamics Lab. Report, AFFDL TR 65-113, 1965

[2] R.E. Bolz and G.L. Tuve, CRC Handbook of Tables for Applied Engineering Science, CRC Press, Boca Raton, FL, 1973.

[3] H. Hwangbo, The Korea domestic communications and broadcasting satellite system, in: AIAA, A Collection of Technical Papers of the 14th International Communication Satellite Systems, Washington, DC, March 22-24, 1992, Vol. 1, pp. 550555.

[4] H. Koguchi and S. Parman, The dynamics of a satellite with flexible solar panels: The vibration of solar panels approached to behave as structures with proportional damping, in: Proc. Int. Session 72nd JSME Spring Annual Meeting, The Japan Society of Mechanical Engineers, Tokyo, 1995, pp. 70-73.

[5] H. Koguchi and S. Parman, Attitude dynamics of a satellite with flexible solar panels based on its nonlinear equations of motion, in: Proc. Int. Session 73rd JSME Spring Annual Meeting, The Japan Society of Mechanical Engineers, Tokyo, 1996, Part VI, pp. 75-78.

[6] H. Koguchi and S. Parman, Attitude dynamics of a satellite with flexible solar panels: Comparison between the nonlinear equations and linearized equations of motion, in: Proc. 3rd Int. Conf. Motion and Vibration Control, K. Nonami and T. Mizuno, eds, The Japan Society of Mechanical Engineers, Tokyo, 1996, Vol. 3, pp. 382-387.

[7] P.W. Likins, Finite element appendage equations for hybrid coordinate dynamic analysis, Int. J. Solids Stuctures 8 (1972), 709-731. 
[8] Q. Liu and B. Wie, Robust time-optimal control of uncertain flexible spacecraft, J. Guidance, Control, and Dynamics 15(3) (1992), 597-604.

[9] P.K. Nguyen and P.C. Hughes, Finite-element analysis of CTSlike flexible spacecraft. UTIAS Report No. 205, 1976.

[10] L.Y. Pao and W.E. Singhose, A comparison of constant and variable amplitude command shaping techniques for vibration reduction, in: IEEE Conf. Control Applications, Albany, NY, Institute of Electrical and Electronics Engineers, New York, 1995, pp. 875-881.

[11] K. Rogers and W.P. Seering, Input shaping for limiting loads and vibration in systems with on-off actuators, in: AIAA Guidance, Navigation, and Control Conf., San Diego, CA, 1996.

[12] T. Singh and S.R. Vadali, Robust time-optimal control: A frequency domain approach, J. Guidance, Control, and Dynamics 17(2) (1994), 346-353.
[13] W. Singhose, W. Seering and N. Singer, Residual vibration reduction using vector diagrams to generate shaped inputs, $J$. Mechanical Design 116 (1994), 654-659.

[14] W. Singhose, S. Derezinski and N. Singer, Extra-insensitive input shapers for controlling flexible spacecraft, J. Guidance, Control, and Dynamics 19(2) (1996), 385-391.

[15] C.J. Swiggert, Shaped torque techniques, J. Guidance and Control 3(5) (1980), 460-467.

[16] S. Timoshenko, D.H. Young and W.Jr. Weaver, The Vibration Problems in Engineering, Wiley, 1974.

[17] B. Wie, R. Sinha and Q. Liu, Robust time-optimal control of uncertain structural dynamic systems, J. Guidance, Control, and Dynamics 15(5) (1993), 980-983.

[18] Zienkiewicz, The Finite Element Method, 3rd edn, McGrawHill, 1977. 

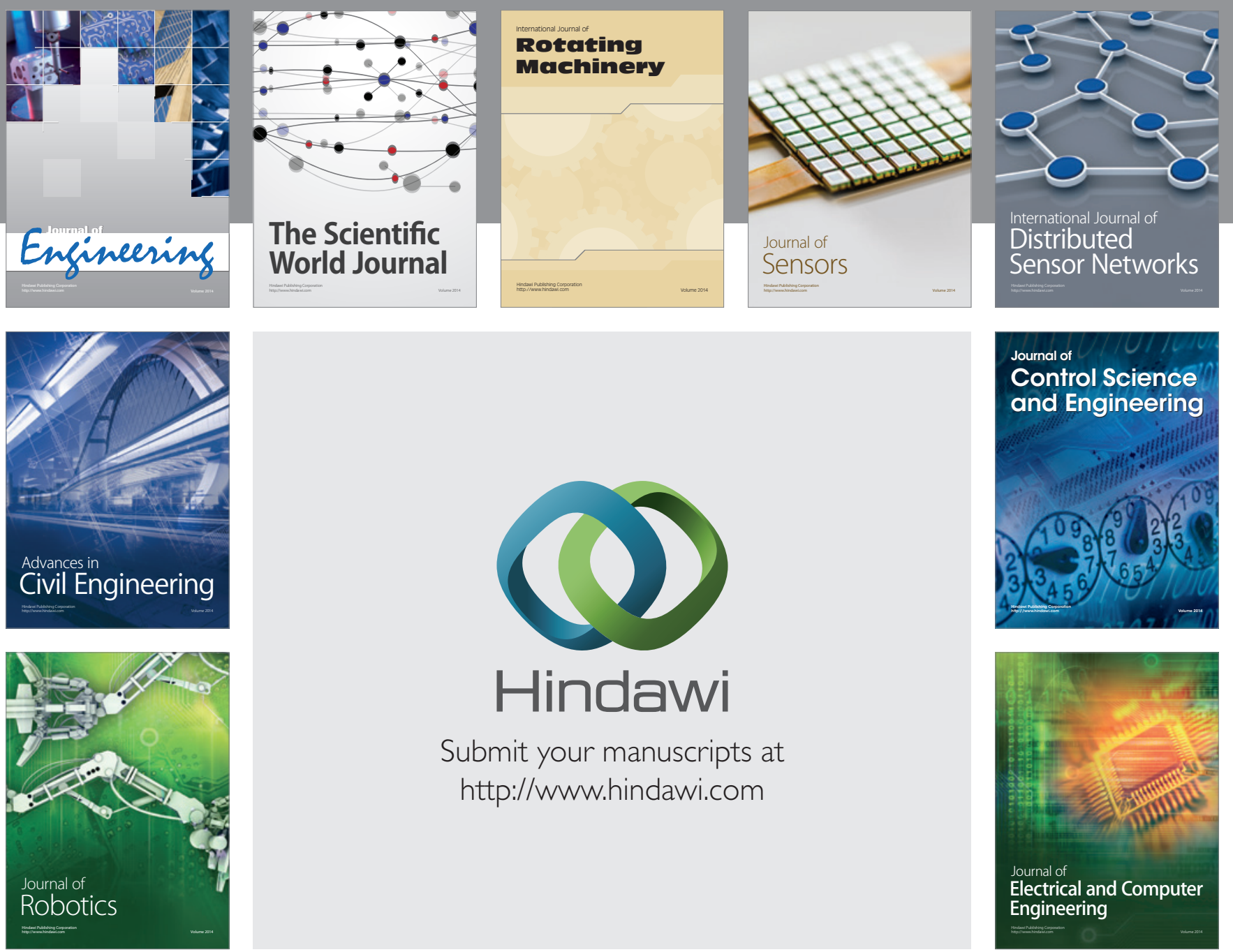

Submit your manuscripts at

http://www.hindawi.com
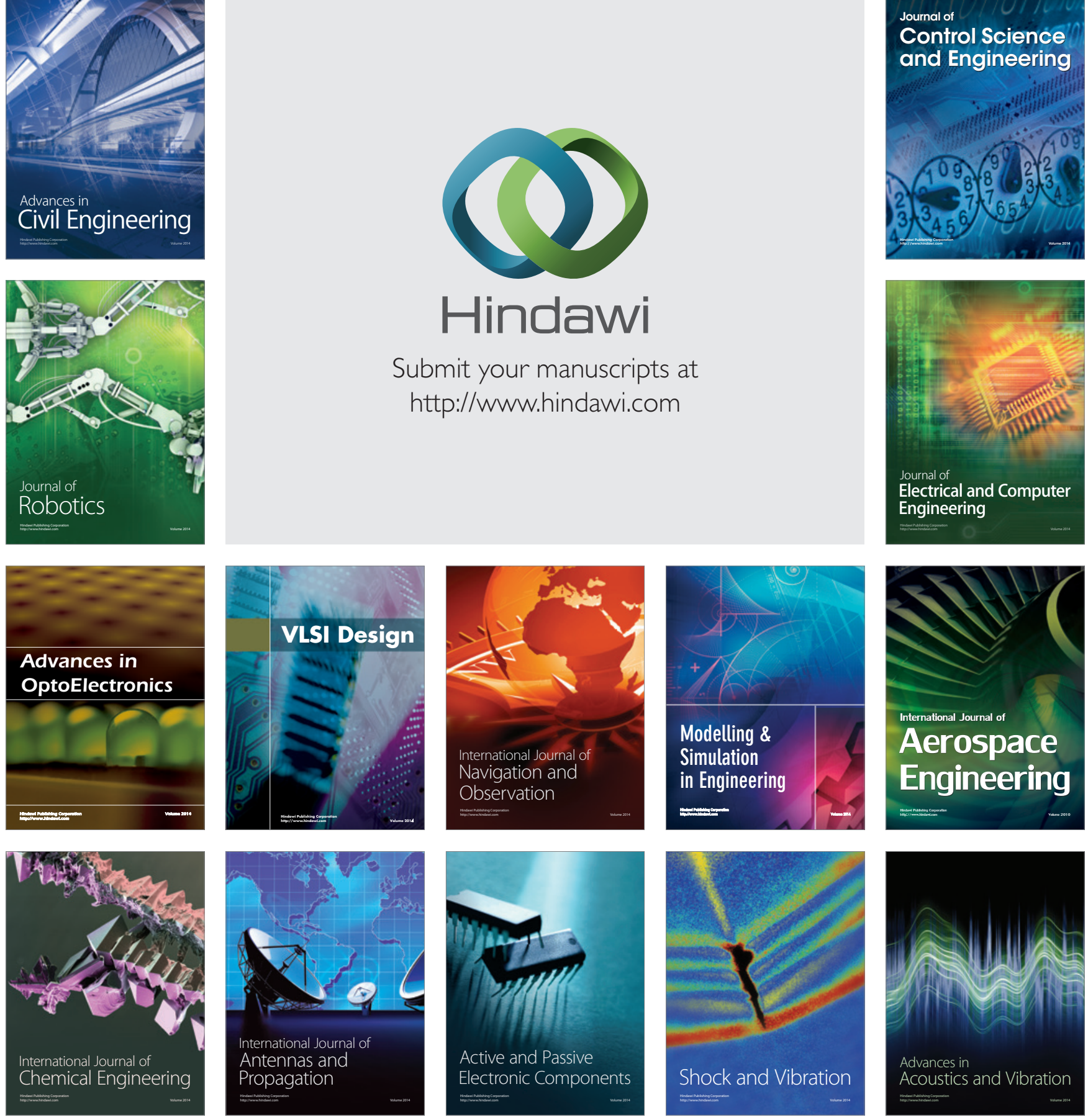